\title{
Distributed Pareto-Optimal Power Control for Utility Maximization in Femtocell Networks
}

\author{
Duy Trong Ngo, Student Member, IEEE, Long Bao Le, Senior Member, IEEE, and Tho Le-Ngoc, Fellow, IEEE
}

\begin{abstract}
This paper proposes two Pareto-optimal power control algorithms for a two-tier network, where newly-deployed femtocell user equipments (FUEs) operate in the licensed spectrum owned by an existing macrocell. Different from homogeneous network settings, the inevitable requirement of robustly protecting the quality-of-service $(\mathrm{QoS})$ of all prioritized macrocell user equipments (MUEs) here lays a major obstacle that hinders the successful application of any available solutions. Directly targeting at this central issue, the first algorithm jointly maximizes the total utility of both user classes. Specifically, we adopt the log-barrier penalty method to effectively enforce the minimum signal-to-interference-plus-noise ratios (SINRs) imposed by the macrocell, paving the way for the adaptation of load-spillage solution framework. On the other hand, the second algorithm is applied to the scenario where only the sum utility of all FUEs needs to be maximized. At optimality, we show that the MUEs' prescribed SINR constraints are met with equality in this case. With the search space for Pareto-optimal SINRs substantially reduced, the second algorithm features scalability, low computational complexity, short converging time, and stable performance. We prove that the two developed algorithms converge to their respective global optima, and more importantly, they can be implemented in a distributive manner at individual links. Effective mechanisms are also available to flexibly designate the access priority to MUEs and FUEs, as well as to fairly share radio resources among users. Numerical results confirm the merits of the devised approaches.
\end{abstract}

Index Terms-Convex optimization, global optimality, heterogeneous network, interference management, Pareto optimality, power control, QoS protection, SINR optimization, utility maximization.

\section{INTRODUCTION}

$\mathbf{F}$ EMTOCELLS have recently emerged as a promising technology to increase wireless network capacity, extend cellular coverage and introduce new services [1], [2]. Femtocell base stations (BSs) are low-power, miniature wireless access points that are typically set at a home and connected to backhaul networks via residential wireline broadband access

Manuscript received August 2, 2011; revised February 21, 2012; accepted August 2, 2012. The associate editor coordinating the review of this paper and approving it for publication is S. Liew.

This work is supported in part by the Natural Sciences and Engineering Research Council of Canada (NSERC) Grants, the Alexander Graham Bell Canada Graduate Scholarship, the McGill Engineering Doctoral Award, and the Clifford Pang Doctoral Fellowship. Part of this paper was presented at the 2011 IEEE International Symposium on Personal, Indoor and Mobile Radio Communications (PIMRC), Toronto, ON, Canada, and the 2012 IEEE Vehicular Technology Conference (VTC-Fall), Quebec City, QC, Canada.

D. T. Ngo and T. Le-Ngoc are with the Department of Electrical and Computer Engineering, McGill University, Montréal, QC, Canada H3A 0E9 (e-mail: duy.ngo@mail.mcgill.ca; tho.le-ngoc@mcgill.ca).

L. B. Le is with the Centre Énergie Matériaux Télécommunications, Institut National de la Recherche Scientifique (INRS-EMT), Université du Québec, Montréal, QC, Canada H5A 1K6 (e-mail: long.le@emt.inrs.ca).

Digital Object Identifier 10.1109/TWC.2012.090312.111454 links, e.g., digital subscriber lines (DSL). Because femtocells can be easily integrated into an existing cellular network infrastructure, their deployment requires low capital expenditures and operating expenses. At the same time, indoor users can enjoy excellent wireless experience thanks to the close proximity with their home femtocell BSs [3].

Since femtocells operate in the licensed spectrum owned by wireless operators and share this spectrum with macrocell networks, limiting the cross-tier interference from FUEs at a macrocell BS becomes an indispensable condition. Due to the random deployment of femtocell BSs, users in a femtocell may also suffer severe interference from nearby femtocells. One of the central research topics is to develop interference management schemes such that the QoS requirements of all MUEs are maintained, while the residual network capacity is optimally exploited by FUEs [4], [5]. Given that only limited signaling information can be exchanged over the backhaul wireline network, it is always desirable in practice to accomplish such optimization by distributed mechanisms.

Using noncooperative game theory, the studies of [6], [7] propose distributed power control schemes for traditional code-division multiple access (CDMA) systems, where individual users select transmit power strategies to maximize their own utility. For femtocell networks, power control games are also formulated and analyzed by [8]-[11]. The work in [12] develops joint power and admission control schemes for distributed interference management in two-tier networks. Specifically, both throughput-power tradeoff optimization and soft QoS provisioning are achieved for FUEs while the predefined SINR targets of all MUEs are always met. In most instances, the underlying games settle at some Nash equilibrium (NE), a stable and predictable state at which no user has any incentive to unilaterally change its power level. Although the achieved NE gives a steady operating point, it does not guarantee to be Pareto-efficient.

A number of pricing schemes are adopted in [13]-[17] to improve the efficiency of the NE. Nevertheless, it remains unclear how far to the global optima of the original problems these solutions actually are. With a novel pricing scheme, the work of [18] shows that the outcome of a non-cooperative power control game in single-cell systems can be a unique and Pareto-efficient NE. Various design goals can also be met by setting dynamic prices for the individual users. In multicell settings where transmit powers of all users need to be jointly optimized across different cells, intercell interference, however, cannot simply be treated as noise, making the solution devised by [18] inapplicable.

In [19], a distributed power control algorithm is proposed 
that enables users to eventually achieve their fixed target SINRs. As long as all the SINR targets are feasible, this iterative algorithm converges to a Pareto-optimal solution at a minimal aggregate transmit power. However, a fixed SINR assignment is certainly not suitable for two-tier networks. Since femtocells typically serve data users, SINRs should instead be adjusted to the extent that the system capacity can still support. A high SINR is translated into better throughput and reliability, whereas a low SINR implies lower data rates.

Under the proposed load-spillage framework, [20] devises distributed solutions to jointly optimize SINRs and powers. While guaranteeing to offer Pareto-optimal SINR assignments, the approach adopted by [20] is directly applicable to homogeneous networks. Herein, there exist no differentiated classes of users with distinct access priority and design specifications. On the contrary, it is imperative to protect the ongoing operation of the preferential MUEs at all times in a twotier heterogeneous network. This critical requirement, indeed, poses a major challenge that hinders the successful application of any available solutions, including [20]. Specifically, the choices of target SINRs available to the lower-tier FUEs in this case are much more limited, which further complicate the Pareto-optimal boundary of the feasible SINR region. As a direct consequence, locating a particular SINR point on such a boundary to optimize some certain system-wide design criteria is by no means a trivial task.

Directly targeting this central issue, this paper attempts to come up with interference management solutions wherein (i) all users attain their respective SINRs that are always optimal in Pareto sense, and (ii) macrocell and femtocell networks have their utilities globally maximized. To handle the above-mentioned QoS requirements of the prioritized MUEs, we first propose the Joint Utility Maximization with macrocell Quality-of-Service guarantee (JUM-QoS) algorithm that maximizes the total utility of both macrocell and femtocell networks. In particular, the minimum SINRs prescribed by the MUEs are effectively enforced with the use of a logbarrier penalty function. After this key step, the Pareto-optimal boundary of the strongly-coupled feasible SINR region is characterized, and the load-spillage framework [20] specifically adapted to find the SINR that approximately maximizes the sum utility. Finally, the global optimum of the original problem is attained by properly tuning the penalty parameter in the proposed penalty approach.

In the specific case where MUEs only need to be assured with some predefined minimum SINRs, we devise the Femtocell Utility Maximization with Macrocell SINR Balancing (FUM-MSB) algorithm. Upon observing the structure of the objective function and the monotonicity of SINR, we confine the Pareto-optimal SINR boundary to a much smaller space. Only then the load-spillage parametrization is applied to the FUEs, whereas the loads of all MUEs are updated according to a newly-developed iterative procedure. Still operating on the Pareto-optimal SINR frontier and retaining the global optimality, this algorithm outperforms the general counterpart JUM-QoS in several important aspects, including scalability, computational complexity, convergence behavior, and stability around the optimum.

It is noteworthy that the proposed JUM-QoS algorithm can also control the access priority of both macrocell and femtocell networks by granting a proper weight to each class of users. In both developed algorithms, the adopted $\alpha$-fair utility function can always be regulated to give different degrees of fairness in allocating radio resources to the individual users. Moreover, our devised schemes can be locally executed, incurring little signaling and information exchange. This feature is particularly attractive in view of practical implementation under the limited backhaul network capacity available for femtocells.

The rest of this paper is organized as follows: Section II presents the system model under investigation and also formulates the design problem. Section III devises the JUMQoS algorithm for the general scenario, where the utilities of both macrocell and femtocell networks are jointly maximized. Section IV proposes the FUM-MSB algorithm for interference management in the special case that the macrocell network only demands to maintain its minimum SINRs. The performance of the two developed schemes is verified by numerical results in Section V. Finally, Section VI concludes the paper.

\section{System Model And PROBlem Formulation}

Consider an uplink scenario in a two-tier wireless network, in which $M$ MUEs establish communication links to its servicing macrocell BS while $K$ FUEs also transmit to their respective femtocell BSs. We assume that all the MUEs and FUEs share the same radio frequency bands by CDMA. To avoid the issues of privacy and security for home (i.e., femtocell) users, closed access mode is considered where the MUEs are not permitted to connect a femtocell BS even if that $\mathrm{BS}$ is within their close range. Also assume that the association of a certain FUE with its own femtocell BS is fixed during the runtime of the underlying power control. Without the loss of generality, denote the set of MUEs and FUEs by $\mathcal{L}_{m}:=\{1, \cdots, M\}$ and $\mathcal{L}_{f}:=\{M+1, \cdots, M+K\}$, respectively. The set of all users is then simply $\mathcal{L}:=\mathcal{L}_{m} \cup \mathcal{L}_{f}$ whose cardinality is $|\mathcal{L}|=M+K$. An example of the system under investigation is illustrated in Fig. 1. It is assumed here that the time scale of network topology changes is negligible compared to that of power adaptation. In addition, data transmission time scale is far shorter than that of the underlying optimization process, which allows any short-term statistical variations to be averaged out (see, e.g., [21]).

Denote by $\sigma_{i}$ the serving BS of user $i \in \mathcal{L}$ (which is either an MUE or an FUE). For brevity, the path between user $i$ and its servicing $\mathrm{BS} \sigma_{i}$ shall be referred to as link $i$. Also, let $\bar{h}_{k, j}$ be the absolute channel gain from user $j$ to BS $k$, and define its corresponding normalization as $h_{k, j}:=\bar{h}_{k, j} / \bar{h}_{\sigma_{j}, j}$. To represent the normalized channel gain from user $j$ to the serving BS $\sigma_{i}$ of user $i$, we define an $(M+K) \times(M+K)$ channel matrix $\mathbf{H}$ whose $(i, j)$-th entry is

$$
H_{i, j}:= \begin{cases}0, & \text { if } i=j, \\ 1, & \text { if } \sigma_{i}=\sigma_{j}, i \neq j, \\ h_{\sigma_{i}, j}, & \text { if } \sigma_{i} \neq \sigma_{j} .\end{cases}
$$

Suppose that user $j$ transmits to its serving $\mathrm{BS} \sigma_{j}$, and let $p^{(j)}$ be the received power at $\sigma_{j}$ by that transmission. Since $\bar{h}_{\sigma_{j}, j}$ is the channel gain from $j$ to $\sigma_{j}$, it is clear that $j$ must have transmitted at a power level $p^{(j)} / \bar{h}_{\sigma_{j}, j}$. At any BS $k$, this signal appears with a power $\bar{h}_{k, j}\left(p^{(j)} / \bar{h}_{\sigma_{j}, j}\right)=h_{k, j} p^{(j)}$. 


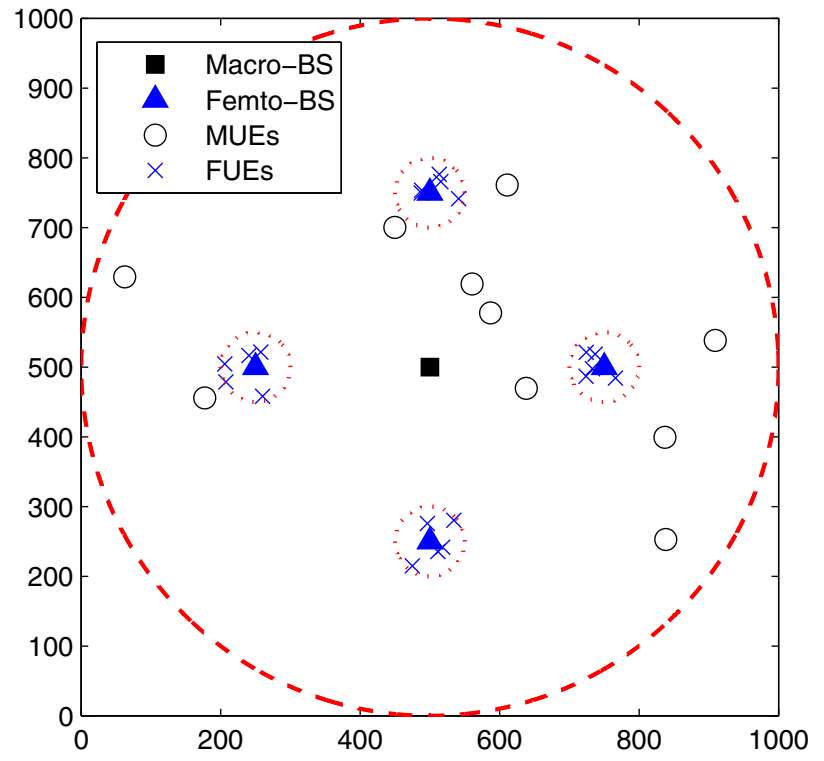

Fig. 1. Network topology and user placement in a two-tier wireless network.

Denote by $\mathcal{S}_{i}$ the set of users whose transmit powers appear as interference to link $i$. If $j \in \mathcal{S}_{i}$, this transmission interferes link $i$ with power $h_{\sigma_{i}, j} p^{(j)}=H_{i, j} p^{(j)}$. The total interference plus noise at $\mathrm{BS} \sigma_{i}$ that serves user $i \in \mathcal{L}$ on link $i$ can be expressed as

$$
q^{(i)}:=\sum_{j \in \mathcal{S}_{i}} H_{i, j} p^{(j)}+\varphi^{(i)}=\sum_{j=1}^{M+K} H_{i, j} p^{(j)}+\varphi^{(i)},
$$

where $\varphi^{(i)}$ is the noise power at the receiving end of link $i$. Throughout this paper, we make a reasonable assumption that $\varphi=\left[\varphi^{(1)}, \cdots, \varphi^{(M+K)}\right]^{T} \neq \mathbf{0}$. In a matrix form, (2) can also be written as

$$
\mathbf{q}=\mathbf{H p}+\varphi
$$

Let $\bar{\gamma}^{(i)}:=G p^{(i)} / q^{(i)}$ denote the received SINR at link $i \in \mathcal{L}$, where $G$ is the system processing gain. For notational convenience, we define the normalized SINR at link $i$ as $\gamma^{(i)}:=\bar{\gamma}^{(i)} / G$. It is then easy to see that

$$
\mathbf{p}=\mathbf{D}(\gamma) \mathbf{q},
$$

where $\mathbf{D}(\gamma):=\operatorname{diag}\left(\gamma^{(1)}, \cdots, \gamma^{(M+K)}\right)$. By substituting (4) to (3) and after some simple algebra, we yield [20]

$$
\begin{aligned}
& \mathbf{q}=\mathbf{H D}(\gamma) \mathbf{q}+\boldsymbol{\varphi} \\
& \mathbf{p}=\mathbf{D}(\gamma) \mathbf{H p}+\mathbf{D}(\gamma) \varphi
\end{aligned}
$$

Since we do not consider totally isolated groups of links that are not interacting with each other, it is practical to assume that both matrices $\mathbf{H D}(\gamma)$ and $\mathbf{D}(\gamma) \mathbf{H}$ are primitive ${ }^{1}$.

The goal of this paper is to devise a jointly optimal power allocation $\mathbf{p}$ and SINR assignment $\gamma$ for the two types of users (i.e., MUEs and FUEs) with different service priorities and design objectives. The prioritized MUEs with higher access rights demand that their ongoing services be, at least, unaffected regardless of any femtocell deployment. A set of

\footnotetext{
${ }^{1} \mathrm{~A}$ non-negative matrix is called primitive if it is irreducible and has only one eigenvalue of maximum modulus [22, Def. 8.5.0].
}

minimum SINRs $\gamma^{\min }=\left[\gamma^{(1) \min }, \cdots, \gamma^{(M) \min }\right]^{T}$ prescribed by the MUEs must therefore be supported in the first place, i.e.,

$$
\gamma^{(i)} \geq \gamma^{(i) \min }, \quad \forall i \in \mathcal{L}_{m},
$$

where $\gamma^{(i) \min }$ is the normalized target SINR corresponding to the actual SINR $\bar{\gamma}^{(i) \min }=G \gamma^{(i) \text { min }}$ required by MUE $i$. Note that a general QoS $\gamma^{\text {min }}$ can be translated to different specific requirements. For instance, a higher value of $\gamma^{(i) \mathrm{min}}$ means that a higher throughput, a lower bit-error rate (BER), and a shorter time delay are guaranteed for MUE $i$.

Our design objective is to maximize the sum utility of all users. Typically an increasing function, the utility $U_{i}\left(\gamma^{(i)}\right)$ represents the value that user $i \in \mathcal{L}$, who is assigned with SINR $\gamma^{(i)}$, contributes to the overall network. The higher the SINR, the greater the contribution. Depending on the type of utility functions, fairness, an important system-wide objective, can also be achieved. Proportional fairness and maxmin fairness are among the most common metrics used in practice to characterize how competing users share system resources. The $\alpha$-fair function proposed by [23] provides a useful means to enforce these two types of fairness, in that it generalizes proportional fairness and includes arbitrarily close approximations of max-min fairness. Specifically, we consider the following utility for user $i \in \mathcal{L}$ :

$$
U_{i}\left(\gamma^{(i)}\right):= \begin{cases}\log \left(\gamma^{(i)}\right), & \text { if } \alpha=1 \\ (1-\alpha)^{-1}\left(\gamma^{(i)}\right)^{1-\alpha}, & \text { if } \alpha \geq 0 \text { and } \alpha \neq 1 .\end{cases}
$$

Here, $\alpha=1$ corresponds to proportional fairness whereas $\alpha \rightarrow \infty$ gives max-min fairness.

Let $\rho(\mathbf{X})$ denote the spectral radius of matrix $\mathbf{X}$, i.e., the maximum modulus eigenvalue of $\mathbf{X}$. Given channel matrix $\mathbf{H}$, the specific value of $\rho(\mathbf{H D}(\gamma))$ indicates whether a certain SINR $\gamma$ is supportable. In particular, it is required by [24] that $\rho(\mathbf{H D}(\gamma))<1$ for the existence of a feasible power vector $\mathbf{p} \succ \mathbf{0}$. In the limit $\rho(\mathbf{H D}(\gamma))=1$, an infinite amount of transmit power would be needed to attain $\gamma$. For $\rho(\operatorname{HD}(\gamma))>1$, the network can be regarded so congested that only removing certain users and/or lowering the SINR targets can help relieve such congestion. Considering a practical non-congested network with attainable target SINRs, we insist that $\rho(\mathbf{H D}(\gamma)) \leq \bar{\rho}$ where $0 \leq \bar{\rho}<1$, for the existence of a feasible solution with $0<p^{(i)}<\infty, \forall i \in \mathcal{L}$.

Given $\bar{\rho} \in[0,1)$, we are interested in the following problem:

$$
\begin{aligned}
\max _{\gamma \in \mathbb{R}_{+}^{M+K}, \mathbf{p} \in \mathbb{R}_{+}^{M+K}} & w_{m} \sum_{i \in \mathcal{L}_{m}} U_{i}\left(\gamma^{(i)}\right)+w_{f} \sum_{i \in \mathcal{L}_{f}} U_{i}\left(\gamma^{(i)}\right) \\
\text { s.t. } & \rho(\mathbf{H D}(\gamma)) \leq \bar{\rho}, \\
& \gamma^{(i)} \geq \gamma^{(i) \min }, \forall i \in \mathcal{L}_{m}
\end{aligned}
$$

where $w_{m} \geq 0$ and $w_{f} \geq 0$ can be used to designate the importance toward the macrocell and femtocell network, respectively. It can be shown that a larger value of $\bar{\rho}$ corresponds to a larger feasible set, and in turn a potentially higher utility. Therefore, it is desirable to choose $\bar{\rho}$ to be as close to 1 as possible while ensuring that $\gamma$ be supportable.

Problem (9) is not convex because the set $\{\gamma \in$ $\left.\mathbb{R}_{+}^{M+K} \mid \rho(\mathbf{H D}(\gamma)) \leq \bar{\rho}\right\}$ is not convex. However, if we let $\boldsymbol{\Gamma}:=\log \gamma$ then its equivalence $\{\boldsymbol{\Gamma} \in$ 
$\left.\mathbb{R}^{M+K} \mid \rho\left(\mathbf{H D}\left(e^{\boldsymbol{\Gamma}}\right)\right) \leq \bar{\rho}\right\}$ is actually a convex set [25]. Through such a change of variable and upon denoting $\Gamma^{(i) \min }:=\log \left(\gamma^{(i) \min }\right)$, the following equivalent problem of (9) is considered instead:

$$
\begin{aligned}
\max _{\boldsymbol{\Gamma} \in \mathbb{R}^{M+K}, \mathbf{p} \in \mathbb{R}_{+}^{M+K}} & w_{m} \sum_{i \in \mathcal{L}_{m}} U_{i}\left(\Gamma^{(i)}\right)+w_{f} \sum_{i \in \mathcal{L}_{f}} U_{i}\left(\Gamma^{(i)}\right) \\
\text { s.t. } & \rho\left(\mathbf{H D}\left(e^{\boldsymbol{\Gamma}}\right)\right) \leq \bar{\rho}, \\
& \Gamma^{(i)} \geq \Gamma^{(i) \min }, \forall i \in \mathcal{L}_{m} .
\end{aligned}
$$

In this case, the utility function becomes

$$
U_{i}\left(\Gamma^{(i)}\right)= \begin{cases}\Gamma^{(i)}, & \text { if } \alpha=1 \\ (1-\alpha)^{-1} e^{(1-\alpha) \Gamma^{(i)}}, & \text { if } \alpha \geq 0 \text { and } \alpha \neq 1,\end{cases}
$$

which is increasing, twice-differentiable and concave with respect to $\Gamma^{(i)}$. Problem (10) is a convex optimization program. However, due to the complicated coupling in the feasible region, centralized algorithms are typically needed to resolve this kind of problem. Given the nature of two-tier networks where central coordination and processing is usually inaccessible, we aim at developing optimal solutions that can be distributively implemented by individual users.

\section{Distributed POWER CONTROL FOR JOINT UTILITY MAXIMIZATION WiTH MACROCELl QOS PROTECTION}

A. Pareto-optimal SINR Boundary and Approximate Solution via Log-barrier Penalty Method

We approach problem (10) by first finding the Paretooptimal boundary $^{2}$ of the feasible SINR region, followed by adapting power to achieve a particular SINR point on that frontier. It is therefore imperative to characterize such a boundary, through which the coupling can be revealed, allowing for the realization of any distributed solutions.

Proposition 1: The Pareto-optimal SINRs for problem (10) lie on the following boundary:

$$
\begin{array}{r}
\partial \mathcal{G}_{\bar{\rho}}:=\left\{\boldsymbol{\Gamma} \in \mathbb{R}^{M+K} \text { s.t. } \rho\left(\mathbf{H D}\left(e^{\boldsymbol{\Gamma}}\right)\right)=\bar{\rho}\right. \\
\text { and } \left.\Gamma^{(i)} \geq \Gamma^{(i) \min }, \forall i \in \mathcal{L}_{m}\right\} .
\end{array}
$$

Proof: The key steps of the proof can be adapted from those of [20, Th. 1] as follows. First, it can be proven that $\partial \rho\left(\mathbf{H D}\left(e^{\boldsymbol{\Gamma}}\right)\right) / \partial \Gamma^{(i)}=v^{(i)} \tilde{q}^{(i)} e^{\Gamma_{i}}, \forall i \in \mathcal{L}$, where $v^{(i)}$ and $\tilde{q}^{(i)}$ respectively are the $i$-th component of the left and the right eigenvectors of $\mathbf{D}\left(e^{\boldsymbol{\Gamma}}\right) \mathbf{H}$ and $\mathbf{H D}\left(e^{\boldsymbol{\Gamma}}\right)$, both associated with the same eigenvalue $\rho\left(\mathbf{D}\left(e^{\boldsymbol{\Gamma}}\right) \mathbf{H}\right)=\rho\left(\mathbf{H D}\left(e^{\boldsymbol{\Gamma}}\right)\right)$ [see Appendix A for a complete derivation]. The properties of primitive matrices imply that $v^{(i)}>0$ and $\tilde{q}^{(i)}>0$ [22, Th. 8.4.4], which means that $\rho\left(\operatorname{HD}\left(e^{\boldsymbol{\Gamma}}\right)\right)$ is increasing with respect to $\Gamma^{(i)}$. Therefore, $\rho\left(\mathbf{H D}\left(e^{\boldsymbol{\Gamma}}\right)\right)=\bar{\rho}$ holds for any $\boldsymbol{\Gamma}$ on the Pareto-optimal SINR boundary.

Prop. 1 indicates that the search space for Pareto-optimal SINR assignments of (10) is $\mathbb{R}^{M+K}$, confined within the surface $\partial \mathcal{G}_{\bar{\rho}}$ specified by $\bar{\rho}$ and $\Gamma^{(i) \min }, \forall i \in \mathcal{L}_{m}$. Consider a simple 3-user network, Fig. 2 illustrates the Pareto-optimal SINR boundaries in both homogeneous and heterogeneous

\footnotetext{
${ }^{2}$ A feasible SINR $\boldsymbol{\Gamma}$ is called Pareto-optimal if it is impossible to increase the SINR of any one link without simultaneously reducing the SINR of some other link.
}

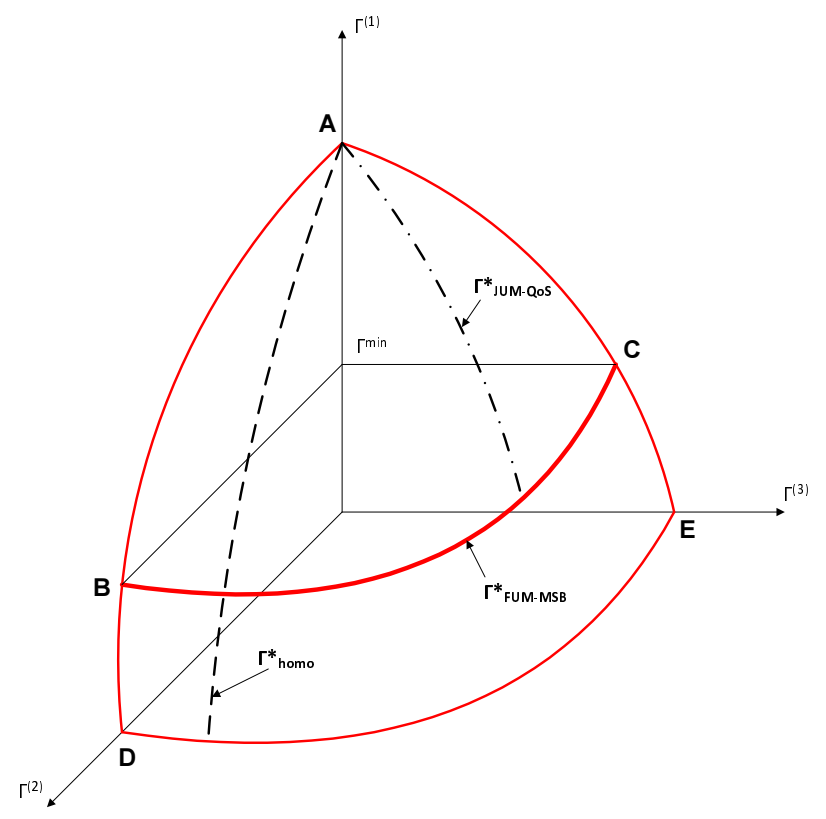

Fig. 2. Pareto-optimal SINR boundary of a network consisting of 1 MUE (i.e., user 1 with $\Gamma^{(1)} \geq \Gamma^{\min }$ ) and 2 FUEs (i.e., users 2 and 3). In homogeneous network settings, $\Gamma_{\text {homo }}^{*}$ lies on the surface ADE. In JUMQoS case, $\Gamma_{\mathrm{JUM}-\mathrm{QoS}}^{*}$ lies on the surface ABC. In FUM-MSB case, $\boldsymbol{\Gamma}_{\mathrm{FUM}-\mathrm{MSB}}^{*}$ lies on the curve connecting $\mathrm{B}$ and $\mathrm{C}$.

scenarios. As can be seen from this example, the optimal SINRs in the heterogeneous case are limited to a smaller region $\mathrm{ABC}$ of the surface $\mathrm{ADE}$ that contains all possible Pareto-optimal SINRs in the homogeneous case. Locating a particular SINR point on the boundary $\mathrm{ABC}$ (i.e., $\partial \mathcal{G}_{\bar{\rho}}$ ) that maximizes the objective of (10) is not trivial at all. The solution in [20], originally developed for homogeneous networks, is not directly applicable here. It might happen that the SINR point given by [20] lies strictly within the surface BCDE, i.e., outside $\partial \mathcal{G}_{\bar{\rho}}$. In this situation, the successful application of the load-spillage approach relies heavily upon how the critical QoS requirements $\Gamma^{(i)} \geq \Gamma^{(i) \min }, \forall i \in \mathcal{L}_{m}$ are managed.

Toward this end, we propose to represent these QoS constraints by the following indicating function:

$$
I_{-}\left(\Gamma^{(i)}\right):= \begin{cases}0, & \text { if } \Gamma^{(i)} \geq \Gamma^{(i) \min } \\ \infty, & \text { otherwise }\end{cases}
$$

for all $i \in \mathcal{L}_{m}$. Problem (10) thus becomes

$$
\begin{aligned}
\max _{\boldsymbol{\Gamma} \in \mathbb{R}^{M+K}, \mathbf{p} \in \mathbb{R}_{+}^{M+K}} & w_{m} \sum_{i \in \mathcal{L}_{m}} U_{i}\left(\Gamma^{(i)}\right)+w_{f} \sum_{i \in \mathcal{L}_{f}} U_{i}\left(\Gamma^{(i)}\right) \\
& -\sum_{i \in \mathcal{L}_{m}} I_{-}\left(\Gamma^{(i)}\right) \\
\text { s.t. } & \rho\left(\mathbf{H D}\left(e^{\boldsymbol{\Gamma}}\right)\right) \leq \bar{\rho} .
\end{aligned}
$$

Because of (13), the objective function in (14) is however not differentiable. Therefore, we approximate $I_{-}\left(\Gamma^{(i)}\right)$ by

$\hat{I}_{-}\left(\Gamma^{(i)}\right):= \begin{cases}-\frac{1}{a} \log \left(\Gamma^{(i)}-\Gamma^{(i) \min }\right), & \text { if } \Gamma^{(i)} \geq \Gamma^{(i) \min }(15) \\ \infty, & \text { otherwise, }\end{cases}$

where $a>0$ is a "penalty factor" used to control the accuracy of the above approximation. In fact, the approximation becomes more accurate as $a$ increases. $\hat{I}_{-}\left(\Gamma^{(i)}\right)$ is 
convex, non-increasing and differentiable, which also implies the concavity of the objective function in (14). Let $\Phi(\boldsymbol{\Gamma}):=-\sum_{i \in \mathcal{L}_{m}} \log \left(\Gamma^{(i)}-\Gamma^{(i) \min }\right)$, whose domain is $\left\{\boldsymbol{\Gamma} \in \mathbb{R}^{M+K} \mid \Gamma^{(i)}>\Gamma^{(i) \min }, \forall i \in \mathcal{L}_{m}\right\}$. The following problem, which approximates (14), can then be considered:

$$
\begin{array}{rl}
\max _{\boldsymbol{\Gamma} \in \mathbb{R}^{M+K}, \mathbf{p} \in \mathbb{R}_{+}^{M+K}} & a\left[w_{m} \sum_{i \in \mathcal{L}_{m}} U_{i}\left(\Gamma^{(i)}\right)\right. \\
& \left.+w_{f} \sum_{i \in \mathcal{L}_{f}} U_{i}\left(\Gamma^{(i)}\right)\right]-\Phi(\boldsymbol{\Gamma}) \\
\text { s.t. } & \rho\left(\mathbf{H D}\left(e^{\boldsymbol{\Gamma}}\right)\right) \leq \bar{\rho} .
\end{array}
$$

With the proposed penalty function $\Phi(\boldsymbol{\Gamma})$, the macrocell SINR constraints are effectively eliminated from the constraint set, leaving the Pareto-optimal SINR surface be simply $\{\boldsymbol{\Gamma} \in$ $\left.\mathbb{R}^{M+K} \mid \rho\left(\mathbf{H D}\left(e^{\boldsymbol{\Gamma}}\right)\right)=\bar{\rho}\right\}$. To distributively realize all points on that surface, we can now make use of the load-spillage framework [20] and parameterize $\Gamma$ through a new variable $\mathbf{s} \succ \mathbf{0}$ such that $\mathbf{s}^{T} \mathbf{H D}\left(e^{\boldsymbol{\Gamma}}\right)=\bar{\rho} \mathbf{s}^{T}$. Let $\mathbf{v}:=\mathbf{H}^{T} \mathbf{s}$, then the resulting SINR

$$
\Gamma^{(i)}=\log \left(\bar{\rho} s^{(i)} / v^{(i)}\right), \forall i \in \mathcal{L}
$$

is indeed Pareto-optimal. It is also clear that $\mathbf{v}$ is the left eigenvector of $\mathbf{D}\left(e^{\boldsymbol{\Gamma}}\right) \mathbf{H}$ with the associated eigenvalue $\bar{\rho}$ because $\mathbf{v}^{T} \mathbf{D}\left(e^{\boldsymbol{\Gamma}}\right) \mathbf{H}=\bar{\rho} \mathbf{v}^{T}$. Here, $\mathbf{s}$ can be interpreted as the "load" on the network to support an SINR $\boldsymbol{\Gamma}$, whereas $\mathbf{v}$ the "spillage," i.e., the potential interference due to $\boldsymbol{\Gamma}$. More specifically, $v^{(i)}=\sum_{j} H_{j, i} s^{(j)}$ represents the effect of interference induced by link $i$ to all other links, weighted by the load $s^{(j)}$ of each link. Responsible for the spillage $v^{(i)}$ to achieve a given SINR $\Gamma^{(i)}$, link $i$ loads the network with $s^{(i)}=v^{(i)} e^{\Gamma^{(i)}} / \bar{\rho}$, i.e., the network is less tolerant by a factor of $s^{(i)}$ to the interference incurred by other links [20].

By fixing $\bar{\rho} \in[0,1)$ and upon applying the above parametrization, (14) can be solved via an equivalent problem in the variable $\mathbf{s}$. The resolution of this new problem can be accomplished by updating $\mathbf{s}$, taking into account the penalty factor $a$ and $\Gamma^{\mathrm{min}}$, as

$$
s^{(i)}[t+1]:=s^{(i)}[t]+\delta \Delta s^{(i)}[t+1], \forall i \in \mathcal{L}
$$

where

$$
\begin{aligned}
& \Delta s^{(i)}[t+1]:= \\
& \quad \frac{w_{m} U_{i}^{\prime}\left(\Gamma^{(i)}\right)}{\bar{\rho} q^{(i)}}+\frac{1}{a \bar{\rho} q^{(i)}\left(\Gamma_{i}-\Gamma_{i}^{\min }\right)}-s^{(i)}[t], i \in \mathcal{L}_{m}
\end{aligned}
$$

and

$$
\Delta s^{(i)}[t+1]:=\frac{w_{f} U_{i}^{\prime}\left(\Gamma^{(i)}\right)}{\bar{\rho} q^{(i)}}-s^{(i)}[t], i \in \mathcal{L}_{f} .
$$

By the similar arguments used in [20], it can be shown that $\nabla f^{T} \Delta \mathbf{s}=(\partial f / \partial \boldsymbol{\Gamma})^{T}(\partial \boldsymbol{\Gamma} / \partial \mathbf{s}) \Delta \mathbf{s}>0, \forall \mathbf{s} \succ \mathbf{0}$ where $f(\mathbf{s})$ denotes the objective function of (16). This means that (19)(20) actually represents an ascent search direction of $f(\mathbf{s})$.

In the log-barrier penalty method, it is imperative to ensure that the conditions $\Gamma^{(i)}>\Gamma^{(i) \text { min }}, \forall i \in \mathcal{L}_{m}$ be always satisfied after every update step. Otherwise, the resulting $\Gamma$ would lie outside the domain of $\Phi(\boldsymbol{\Gamma})$, making the objective function of (16) unbounded below. To this end, as long as $\Gamma^{(i)}$ is not strictly greater than $\Gamma^{(i) \min }$ for any $i \in \mathcal{L}_{m}$, we propose to scale the step size $\delta$ in (18) as $\delta:=b \delta$ where $0<b<1$.

Proposition 2: As $\bar{\rho} \rightarrow 1$, the update of $\mathbf{s}$ in (18) allows the global optimum of the approximate problem (16) to be found.

Proof: Because problem (16) is convex, the idea of the proof is quite standard, i.e., writing down the Karush-KuhnTucker (KKT) conditions [26] as well as the convergence property of the load vector $\mathrm{s}$, and observing that these two sets of equations are actually identical. Note that although [20] has also taken a similar approach, the update of $\mathbf{s}$ is very different in our case since we have accounted for the penalty factor as well as the minimum SINR requirements of the MUEs.

The Lagrangian of (16) is defined as

$$
\begin{array}{r}
\mathcal{L}(\boldsymbol{\Gamma}, \mu):=a\left[w_{m} \sum_{i \in \mathcal{L}_{m}} U_{i}\left(\Gamma^{(i)}\right)+w_{f} \sum_{i \in \mathcal{L}_{f}} U_{i}\left(\Gamma^{(i)}\right)\right] \\
-\Phi(\boldsymbol{\Gamma})-\mu\left[\rho\left(\mathbf{H D}\left(e^{\boldsymbol{\Gamma}}\right)\right)-\bar{\rho}\right], \quad
\end{array}
$$

with multiplier $\mu \geq 0$. It follows from the KKT conditions that $\nabla \mathcal{L}(\boldsymbol{\Gamma}, \mu)=0$, which is equivalent to

$$
\begin{array}{r}
a w_{m} U_{i}^{\prime}\left(\Gamma^{(i)}\right)+\frac{1}{\Gamma^{(i)}-\Gamma^{(i) \min }}=\mu v^{(i)} \tilde{q}^{(i)} e^{\Gamma^{(i)}}, i \in \mathcal{L}_{m} \\
a w_{f} U_{i}^{\prime}\left(\Gamma^{(i)}\right)=\mu v^{(i)} \tilde{q}^{(i)} e^{\Gamma^{(i)}}, i \in \mathcal{L}_{f}
\end{array}
$$

where $\mathbf{v}=\left[v^{(i)}\right]$ is the left eigenvector of $\mathbf{D}\left(e^{\boldsymbol{\Gamma}}\right) \mathbf{H}$ and $\tilde{\mathbf{q}}=$ $\left[\tilde{q}^{(i)}\right]$ the right eigenvector of $\operatorname{HD}\left(e^{\Gamma}\right)$ (normalized such that $\mathbf{s}^{T} \tilde{\mathbf{q}}=1$ ), both associated with eigenvalue $\bar{\rho}$.

On the other hand, at the point of convergence $\mathbf{s}^{*}$, we have that $\Delta \mathbf{s}^{*}=0$. From (19)-(20), it is clear to see that

$$
\begin{aligned}
a w_{m} U_{i}^{\prime}\left(\Gamma^{(i) *}\right)+\frac{1}{\Gamma^{(i) *}-\Gamma^{(i) \min }} & =a \bar{\rho} q^{(i) *} s^{(i) *}, i \in \mathcal{L}_{m} \\
a w_{f} U_{i}^{\prime}\left(\Gamma^{(i) *}\right) & =a \bar{\rho} q^{(i) *} s^{(i) *}, i \in \mathcal{L}_{f} .
\end{aligned}
$$

By [27], it can be shown that

$$
\left[\mathbf{I}-\mathbf{H D}\left(e^{\boldsymbol{\Gamma}}\right)\right]^{-1} \rightarrow \frac{1}{1-\bar{\rho}}\left[\tilde{\mathbf{q}} /\left(\mathbf{s}^{T} \boldsymbol{\varphi}\right)\right]\left[\mathbf{s}^{T} /\left(\mathbf{s}^{T} \boldsymbol{\varphi}\right)\right],
$$

as $\bar{\rho} \rightarrow 1$. Furthermore, some simple manipulation of (5) gives

$$
\mathbf{q}=\left[\mathbf{I}-\mathbf{H D}\left(e^{\boldsymbol{\Gamma}}\right)\right]^{-1} \boldsymbol{\varphi}
$$

It follows that

$$
q^{(i) *} \rightarrow \tilde{q}^{(i)} /\left[(1-\bar{\rho}) \mathbf{s}^{* T} \boldsymbol{\varphi}\right]
$$

as $\bar{\rho} \rightarrow 1$. Upon noting that $\bar{\rho} s^{(i) *}=e^{\Gamma^{(i) *}} v^{(i) *},(24)-(25)$ are exactly (22)-(23) for $\boldsymbol{\Gamma}=\boldsymbol{\Gamma}^{*}$ and $\mu=a /\left[(1-\bar{\rho}) \mathbf{s}^{* T} \boldsymbol{\varphi}\right]>0$. Since (16) is a convex optimization problem, any point that satisfies the KKT conditions is indeed its globally optimal solution.

With $\mathbf{s}^{*}$ known and upon recalling that $\mathbf{v}^{*}=\mathbf{H}^{T} \mathbf{s}^{*}$, the optimal SINR assignment $\boldsymbol{\Gamma}^{*}$ is determined according to (17). By Foschini-Miljanic's algorithm [19], the power allocation $\mathbf{p}^{*}$ that achieves $\boldsymbol{\Gamma}^{*}$ can be found. Indeed, $\left(\boldsymbol{\Gamma}^{*}, \mathbf{p}^{*}\right)$ gives the global optimum of problem (16). 


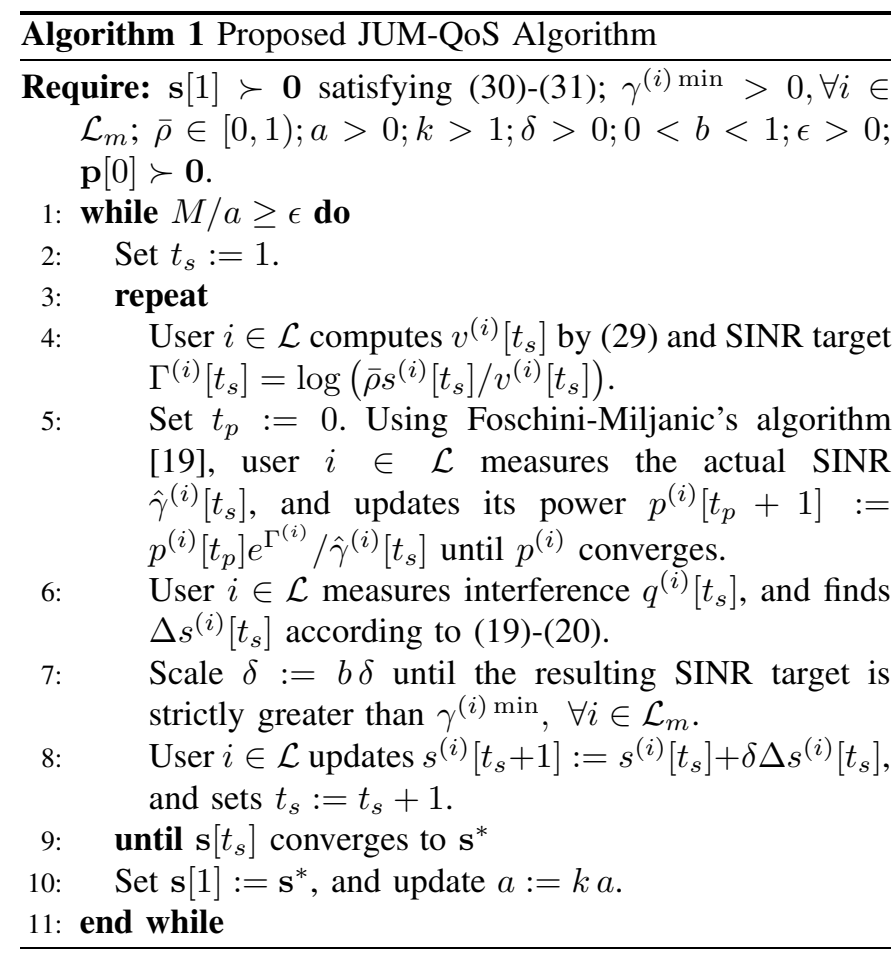

\section{B. Proposed Distributed Algorithm for Globally Maximized Joint Utility}

We present in Alg. 1 the Joint Utility Maximization with macrocell Quality-of-Service guarantee (JUM-QoS) algorithm to solve problem (10). Recall that resolving (16) only gives an approximate solution to problem (14), and in turn (10). Once problem (16) has been resolved, control parameter $a$ needs to be regulated accordingly to make the approximation more accurate. Specifically, there are two levels of execution in this algorithm: the outer loop is to update $a$ whereas the inner loop to find an optimal solution to the approximate problem (16). The resulting $\mathbf{s}$ of the current inner loop will be used in the next iteration of the outer loop.

Importantly enough, the proposed solution can be distributively implemented at each individual link with limited information being exchanged, either by means of wireless broadcasting or over the available backhaul networks (e.g., DSL links). By assuming that the channel gains between the downlink and the uplink are identical, and upon noticing that

$$
\begin{aligned}
v^{(i)} & =\sum_{j \in \mathcal{L}} H_{j, i} s^{(j)} \\
& =\sum_{j \neq i, \sigma_{j}=\sigma_{i}} s^{(j)}+\sum_{l \neq \sigma_{i}} h_{l, i} \sum_{j, \sigma_{j}=l} s^{(j)},
\end{aligned}
$$

the value of $v^{(i)}$ in Step 4 can be computed and managed solely by user $i \in \mathcal{L}$. Specifically, we require each BS $l$ to broadcast the quantity $\sum_{j, \sigma_{j}=l} s^{(j)}$ at a constant power. This permits user $i$ to also measure all the link gains $h_{l, i}=h_{i, l}$ required for the calculation of $\sum_{l \neq \sigma_{i}} h_{l, i} \sum_{j, \sigma_{j}=l} s^{(j)}$.

In Step 7, to check the feasibility of the resulting target SINR $\tilde{\Gamma}_{i}=\log \left(\bar{\rho} \tilde{s}^{(i)} / \tilde{v}^{(i)}\right)$ associated with the search direction $\Delta s^{(i)}$, each user $i \in \mathcal{L}$ computes $\tilde{s}^{(i)}:=s^{(i)}\left[t_{s}\right]+$ $\delta \Delta s^{(i)}\left[t_{s}\right]$, and subsequently $\tilde{v}^{(i)}$ as a function of $\tilde{\mathbf{s}}$ [similar to (29)]. With channel gains $h_{l, i}=h_{i, l}$ already known, the computation of $\tilde{v}^{(i)}$ only requires $\tilde{s}^{(i)}$ to be exchanged, e.g., over backhaul links. It should also be noted that we need to initialize the algorithm with a strictly feasible solution to ensure that $\Gamma^{(i)}>\Gamma^{(i) \min }, \forall i \in \mathcal{L}_{m}$. Since $\Gamma^{(i)}=\log \left(\bar{\rho} s^{(i)} / v^{(i)}\right)$ and $v^{(i)}=\sum_{i \in \mathcal{L}} H_{j, i} s^{(j)}$, this requirement corresponds to solving the following set of linear inequalities:

$$
\begin{aligned}
\bar{\rho} s^{(i)}-\sum_{j \in \mathcal{L} \backslash\{i\}} e^{\Gamma^{(i) \min } H_{j, i} s^{(j)}}>0, \forall i \in \mathcal{L}_{m} \\
s^{(i)}>0, \forall i \in \mathcal{L}_{f} .
\end{aligned}
$$

Theorem 1: As $\bar{\rho} \rightarrow 1$, the proposed JUM-QoS algorithm converges to the global optimum of (10).

Proof: Given $\bar{\rho} \rightarrow 1$, let $\Gamma_{a}^{*}$ be an optimal solution of (16). It follows that $\Gamma_{a}^{(i) *}>\Gamma^{(i) \min }, \forall i \in \mathcal{L}_{m}$ (strictly feasible), and that $\rho\left(\mathbf{H D}\left(e^{\boldsymbol{\Gamma}_{a}^{*}}\right)\right)=\bar{\rho}$ (operating on the Paretooptimal boundary of SINR). With $\mu \in \mathbb{R}$, the stationarity condition of (16) can be expressed as

$$
\begin{aligned}
a w_{m} & \sum_{i \in \mathcal{L}_{m}} U_{i}^{\prime}\left(\Gamma_{a}^{(i) *}\right)+\frac{1}{\Gamma_{a}^{(i) *}-\Gamma^{(i) \min }} \\
& -\mu \tilde{q}^{(i)}\left(\Gamma_{a}^{(i) *}\right) v^{(i)}\left(\Gamma_{a}^{(i) *}\right) e^{\Gamma_{a}^{(i) *}}=0,
\end{aligned}
$$

for all $i \in \mathcal{L}_{m}$, and

$$
a w_{f} \sum_{i \in \mathcal{L}_{f}} U_{i}^{\prime}\left(\Gamma_{a}^{(i) *}\right)-\mu \tilde{q}^{(i)}\left(\Gamma_{a}^{(i) *}\right) v^{(i)}\left(\Gamma_{a}^{(i) *}\right) e^{\Gamma_{a}^{(i) *}}=0,
$$

for all $i \in \mathcal{L}_{f}$.

Now let $\lambda_{i}^{*}:=1 /\left[a\left(\Gamma_{a}^{(i) *}-\Gamma^{(i) \min }\right)\right]>0, \forall i \in \mathcal{L}_{m}$, and $\mu^{*}:=\mu / a$. It follows that

$$
\begin{aligned}
w_{m} & \sum_{i \in \mathcal{L}_{m}} U_{i}^{\prime}\left(\Gamma_{a}^{(i) *}\right)+\lambda_{i}^{*} \\
& -\mu^{*} \tilde{q}^{(i)}\left(\Gamma_{a}^{(i) *}\right) v^{(i)}\left(\Gamma_{a}^{(i) *}\right) e^{\Gamma_{a}^{(i) *}}=0,
\end{aligned}
$$

for all $i \in \mathcal{L}_{m}$, and

$$
w_{f} \sum_{i \in \mathcal{L}_{f}} U_{i}^{\prime}\left(\Gamma_{a}^{(i) *}\right)-\mu^{*} \tilde{q}^{(i)}\left(\Gamma_{a}^{(i) *}\right) v^{(i)}\left(\Gamma_{a}^{(i) *}\right) e^{\Gamma_{a}^{(i) *}}=0,
$$

for all $i \in \mathcal{L}_{f}$. This means that $\Gamma_{a}^{*}$ maximizes the Lagrangian

$$
\begin{aligned}
& \mathcal{L}(\boldsymbol{\Gamma}, \boldsymbol{\lambda}, \xi):=w_{m} \sum_{i \in \mathcal{L}_{m}} U_{i}\left(\Gamma^{(i)}\right)+w_{f} \sum_{i \in \mathcal{L}_{f}} U_{i}\left(\Gamma^{(i)}\right) \\
& +\sum_{i \in \mathcal{L}_{m}} \lambda_{i}\left(\Gamma^{(i)}-\Gamma^{(i) \min }\right)-\xi\left[\rho\left(\mathbf{H D}\left(e^{\boldsymbol{\Gamma}}\right)\right)-\bar{\rho}\right]
\end{aligned}
$$

of problem (10) for $\lambda_{i}=\lambda_{i}^{*}>0, \forall i \in \mathcal{L}_{m}$ and $\xi=\mu^{*}$. From this, it is apparent that $\left(\boldsymbol{\lambda}^{*}, \mu^{*}\right)$ is a dual feasible pair of (10). Therefore, the dual function $g\left(\boldsymbol{\lambda}^{*}, \mu^{*}\right)$ is finite, and also

$$
\begin{aligned}
& g\left(\boldsymbol{\lambda}^{*}, \mu^{*}\right)=w_{m} \sum_{i \in \mathcal{L}_{m}} U_{i}\left(\Gamma_{a}^{(i) *}\right)+w_{f} \sum_{i \in \mathcal{L}_{f}} U_{i}\left(\Gamma_{a}^{(i) *}\right) \\
& +\sum_{i \in \mathcal{L}_{m}} \lambda_{i}^{*}\left(\Gamma_{a}^{(i) *}-\Gamma^{(i) \min }\right)-\mu^{*}\left[\rho\left(\mathbf{H D}\left(e^{\boldsymbol{\Gamma}_{a}^{*}}\right)\right)-\bar{\rho}\right] \\
& =w_{m} \sum_{i \in \mathcal{L}_{m}} U_{i}\left(\Gamma_{a}^{(i) *}\right)+w_{f} \sum_{i \in \mathcal{L}_{f}} U_{i}\left(\Gamma_{a}^{(i) *}\right)+\frac{M}{a},
\end{aligned}
$$

where recall that $M=\left|\mathcal{L}_{m}\right|$ is the total number of MUEs. 
Denote the (primal) optimal value of (10) as $U^{*}:=$ $\min _{\boldsymbol{\lambda}^{*} \succ \mathbf{0}, \mu^{*}} g\left(\boldsymbol{\lambda}^{*}, \mu^{*}\right)$. From (37),

$$
U^{*}-M / a \leq w_{m} \sum_{i \in \mathcal{L}_{m}} U_{i}\left(\Gamma_{a}^{(i) *}\right)+w_{f} \sum_{i \in \mathcal{L}_{f}} U_{i}\left(\Gamma_{a}^{(i) *}\right) .
$$

It is also true that

$$
w_{m} \sum_{i \in \mathcal{L}_{m}} U_{i}\left(\Gamma_{a}^{(i) *}\right)+w_{f} \sum_{i \in \mathcal{L}_{f}} U_{i}\left(\Gamma_{a}^{(i) *}\right) \leq U^{*}
$$

From (38) and (39), it is clear that $\Gamma_{a}^{*}$ approaches the globally optimal solution of the original problem (10) as $a \rightarrow \infty$. At which point, $U^{*}$ is attained.

\section{Distributed Power Control for Femtocell UTILITY MAXIMIZATION AND MACROCELL SINR BALANCING}

The JUM-QoS algorithm proposed in the previous section is applicable to a general scenario with the utilities of both macrocell and femtocell networks jointly optimized. In this case, it is noteworthy that the search space for Pareto-optimal SINRs always spans the entire $M+K$ dimensions. Consider a scenario in which MUEs do not require to maximize any utility, rather only their predefined minimum SINRs are to be protected. A typical example is a macrocell network that mainly serves voice users coexists with a data-serviced femtocell network. Specifically, this instance of problem corresponds to having $w_{m}=0$ in the formulation (10). For notational convenience and without the loss of generality, we let $w_{f}=1$. Then, problem (10) is reduced to:

$$
\begin{aligned}
\max _{\boldsymbol{\Gamma} \in \mathbb{R}^{M+K}, \mathbf{p} \in \mathbb{R}_{+}^{M+K}} & \sum_{i \in \mathcal{L}_{f}} U_{i}\left(\Gamma^{(i)}\right) \\
\text { s.t. } & \rho\left(\mathbf{H D}\left(e^{\boldsymbol{\Gamma}}\right)\right) \leq \bar{\rho}, \\
& \Gamma^{(i)} \geq \Gamma^{(i) \min }, \forall i \in \mathcal{L}_{m} .
\end{aligned}
$$

Upon observing the structure of the objective function in (40) and the monotonicity of SINR, it is shown in the sequel that the Pareto-optimal boundary of the feasible SINR region is confined to a much smaller dimension. This property indeed gives rise to an algorithm that is more efficient than the general JUM-QoS solution.

\section{A. Distributed Pareto-optimal SINR Assignment}

We perform the following matrix and vector partitions: $\mathbf{p}=$ $\left[\mathbf{p}_{m}^{T}, \mathbf{p}_{f}^{T}\right]^{T} ; \mathbf{q}=\left[\mathbf{q}_{m}^{T}, \mathbf{q}_{f}^{T}\right]^{T} ; \boldsymbol{\Gamma}=\left[\boldsymbol{\Gamma}_{m}^{T}, \boldsymbol{\Gamma}_{f}^{T}\right]^{T} ; \boldsymbol{\varphi}=\left[\boldsymbol{\varphi}_{m}^{T}, \boldsymbol{\varphi}_{f}^{T}\right]^{T} ;$ and $\mathbf{H}=\left[\begin{array}{ll}\mathbf{H}_{11} & \mathbf{H}_{12} \\ \mathbf{H}_{21} & \mathbf{H}_{22}\end{array}\right]$, where $\mathbf{q}_{m}, \mathbf{p}_{m}, \boldsymbol{\varphi}_{m} \in \mathbb{R}_{+}^{M}, \boldsymbol{\Gamma}_{m} \in$ $\mathbb{R}^{M} ; \mathbf{q}_{f}, \mathbf{p}_{f}, \boldsymbol{\varphi}_{f} \in \mathbb{R}_{+}^{K}, \boldsymbol{\Gamma}_{f} \in \mathbb{R}^{K} ; \mathbf{H}_{11} \in \mathbb{R}_{+}^{M \times M}, \mathbf{H}_{12} \in$ $\mathbb{R}_{+}^{M \times K}, \mathbf{H}_{21} \in \mathbb{R}_{+}^{K \times M}$, and $\mathbf{H}_{22} \in \mathbb{R}_{+}^{K \times K}$. For the ease of reference, we also let $\boldsymbol{\Gamma}_{m}^{\min }:=\boldsymbol{\Gamma}^{\mathrm{min}}$.

Proposition 3: The optimal solution of (40) lies on the following boundary:

$$
\begin{aligned}
& \partial \mathcal{F}_{\bar{\rho}}:=\left\{\boldsymbol{\Gamma}=\left[\boldsymbol{\Gamma}_{m} ; \boldsymbol{\Gamma}_{f}\right] ; \boldsymbol{\Gamma}_{m} \in \mathbb{R}^{M}, \boldsymbol{\Gamma}_{f} \in \mathbb{R}^{K}\right. \\
&\text { s.t. } \left.\rho\left(\operatorname{HD}\left(e^{\boldsymbol{\Gamma}}\right)\right)=\bar{\rho} \text { and } \boldsymbol{\Gamma}_{m}=\boldsymbol{\Gamma}_{m}^{\text {min }}\right\}
\end{aligned}
$$

Proof: Suppose that at optimality, there is some $\Gamma_{m}^{(i)}$ such that $\Gamma_{m}^{(i)}>\Gamma_{m}^{(i) \min }$. Since $\Gamma_{m}^{(i)}=\log \left(p_{m}^{(i)} / q_{m}^{(i)}\right)$, one can reduce $p_{m}^{(i)}$ to have $\Gamma_{m}^{(i)}=\Gamma_{m}^{(i) \min }$ without violating the constraint. On the other hand, such a reduction in power results in a lesser amount of interference perceived by all the FUEs. Due to the monotonic property, this implies an increase in $\sum_{i \in \mathcal{L}_{f}} U_{i}\left(\Gamma^{(i)}\right)$, and hence contradicts the assumption of optimality. Therefore, $\boldsymbol{\Gamma}_{m}=\boldsymbol{\Gamma}_{m}^{\mathrm{min}}$ holds. Moreover, it can be proven that $\rho\left(\mathbf{H D}\left(e^{\boldsymbol{\Gamma}}\right)\right)=\bar{\rho}$ at the optimum [see Prop. 1]. This completes the proof.

Prop. 3 implies that the search space for Pareto-optimal SINRs in this case is reduced to simply $\mathbb{R}^{K}$. An example of the above-derived boundary $\partial \mathcal{F}_{\bar{\rho}}$ is illustrated in Fig. 2, where the Pareto-optimal SINRs of a 3-user network lie within the curve connecting the two points B and C. To unveil the complicated coupling between $\boldsymbol{\Gamma}_{m}$ and $\boldsymbol{\Gamma}_{f}$ in the relation $\rho\left(\operatorname{HD}\left(e^{\boldsymbol{\Gamma}}\right)\right)=\bar{\rho}$, the following result is now in order.

Proposition 4: Suppose that we are operating on $\partial \mathcal{F}_{\bar{\rho}}$ and that $\rho\left(\mathbf{H}_{11} \mathbf{D}\left(e^{\boldsymbol{\Gamma}_{m}^{\min }}\right)\right)<\bar{\rho}$. Then, $\rho\left(\mathbf{H D}\left(e^{\boldsymbol{\Gamma}}\right)\right)=$ $\rho\left(\mathbf{F D}\left(e^{\boldsymbol{\Gamma}_{f}}\right)\right)$ holds, where $\mathbf{F}$ is a positive matrix defined as

$$
\mathbf{F}:=\mathbf{H}_{21} \mathbf{D}\left(e^{\boldsymbol{\Gamma}_{m}^{\mathrm{min}}}\right)\left[\bar{\rho} \mathbf{I}_{M}-\mathbf{H}_{11} \mathbf{D}\left(e^{\boldsymbol{\Gamma}_{m}^{\mathrm{min}}}\right)\right]^{-1} \mathbf{H}_{12}+\mathbf{H}_{22} \cdot(42)
$$

Proof: The proof is given in Appendix B.

The assumption $\rho\left(\mathbf{H}_{11} \mathbf{D}\left(e^{\boldsymbol{\Gamma}_{m}^{\min }}\right)\right)<\bar{\rho}$ in Prop. 4 can be justified by first noting that the channel matrix $\mathbf{H}$ is reduced to simply $\mathbf{H}_{11}$ if there is no femtocell deployed, and then applying the condition for the existence of a feasible power vector $\mathbf{p}_{m}=\left[p^{(1)}, \cdots, p^{(M)}\right]^{T} \succ \mathbf{0}$ in that case [24]. Essentially, Props. 3 and 4 characterize the following Paretooptimal SINR boundary:

$$
\begin{aligned}
\partial \mathcal{F}_{\bar{\rho}}=\left\{\boldsymbol{\Gamma}=\left[\boldsymbol{\Gamma}_{m} ; \boldsymbol{\Gamma}_{f}\right] ; \boldsymbol{\Gamma}_{m} \in \mathbb{R}^{M}, \boldsymbol{\Gamma}_{f} \in \mathbb{R}^{K}\right. \\
\text { s.t. } \left.\rho\left(\mathbf{F D}\left(e^{\boldsymbol{\Gamma}_{f}}\right)\right)=\bar{\rho} \text { and } \boldsymbol{\Gamma}_{m}=\boldsymbol{\Gamma}_{m}^{\min }\right\}
\end{aligned}
$$

of problem (40). For every point on $\partial \mathcal{F}_{\bar{\rho}}$, it is impossible to increase the SINR of any one femto link without simultaneously reducing the SINR of some other femto links.

The finding of $\mathbf{F}$ in Prop. 4 also reveals that the performance of the FUEs depends not only on the structure of the femtocell network (as reflected in $\mathbf{H}_{22}$ ), but also on the interaction between themselves with the MUEs (as represented by $\mathbf{H}_{21}$ and $\mathbf{H}_{12}$ ). Moreover, the existence of $\mathbf{F}$ is conditional upon the particular values of $\mathbf{H}_{11}$ and $\boldsymbol{\Gamma}_{m}^{\min }$, i.e., $\rho\left(\mathbf{H}_{11} \mathbf{D}\left(e^{\boldsymbol{\Gamma}_{m}^{\min }}\right)\right)<\bar{\rho}$. It is somewhat an expected result because MUEs have an absolutely higher priority in accessing the system resource. Such a condition also confirms that FUEs can attain their Pareto-optimal SINRs only if the performance of MUEs is, at least, unaffected.

The fact that $\mathbf{F}$ is a positive matrix is critical, since it paves the way to adapt the load-spillage parametrization [20] to find all points on $\partial \mathcal{F}_{\bar{\rho}}$. Nevertheless, it is important to point out here that thanks to Props. 3 and 4, one has to only deal with matrix $\mathbf{F}$ in a $K$-dimensional space instead of the original $(M+K) \times(M+K)$ channel matrix $\mathbf{H}$. Also note that $\mathbf{F}$ does not need to be primitive in the following result, unlike the strict condition on completely connected (i.e., primitive) matrices specifically required by [20].

Proposition 5: For a fixed $\boldsymbol{\Gamma}_{m}^{\mathrm{min}}$, an SINR vector $\boldsymbol{\Gamma}=$ $\left[\boldsymbol{\Gamma}_{m} ; \boldsymbol{\Gamma}_{f}\right]$ lies on the boundary $\partial \mathcal{F}_{\bar{\rho}}$ defined in (43) if and 
only if there exist $\mathbf{s}_{f} \succ \mathbf{0}$ in $\mathbb{R}^{K}$ and $\bar{\rho} \in[0,1)$ such that

$$
\begin{aligned}
\boldsymbol{\Gamma}_{m} & =\boldsymbol{\Gamma}_{m}^{\min }, \\
\mathbf{s}_{f}^{T} \mathbf{F D}\left(e^{\boldsymbol{\Gamma}_{f}}\right) & =\bar{\rho} \mathbf{s}_{f}^{T} .
\end{aligned}
$$

Proof: Similar to that of [20, Lem. 3], the idea of this proof is based on Perron's theorem [22, Th. 8.2.11]. If there is some $\mathbf{s}_{f} \succ \mathbf{0}$ and $\bar{\rho} \in[0,1)$ satisfying (45), then $\mathbf{s}_{f}$ is a positive left eigenvector, associated with eigenvalue $\bar{\rho}$, of the positive matrix $\mathbf{F D}\left(e^{\boldsymbol{\Gamma}_{f}}\right)$. By Perron's theorem, $\bar{\rho}$ is a unique positive eigenvalue with maximum modulus, i.e., $\bar{\rho}=\rho\left(\mathbf{F D}\left(e^{\boldsymbol{\Gamma}_{f}}\right)\right)$. Along with $\boldsymbol{\Gamma}_{m}=\boldsymbol{\Gamma}_{m}^{\min }$ in (44), the corresponding $\boldsymbol{\Gamma}$ is on $\partial \mathcal{F}_{\bar{\rho}}$.

Conversely, if $\boldsymbol{\Gamma} \in \partial \mathcal{F}_{\bar{\rho}}$ then $\boldsymbol{\Gamma}_{m}=\boldsymbol{\Gamma}_{m}^{\min }$ and $\rho\left(\mathbf{F D}\left(e^{\boldsymbol{\Gamma}_{f}}\right)\right)=\bar{\rho}<1$. Let $\mathbf{s}_{f}$ be the left eigenvector associated with eigenvalue $\bar{\rho}$. Again, by Perron's theorem, $\mathbf{s}_{f} \succ \mathbf{0}$ and $\bar{\rho}>0$ since $\mathbf{F D}\left(e^{\boldsymbol{\Gamma}_{f}}\right)$ is a positive matrix.

Using Prop. 5, we can now parameterize all $\boldsymbol{\Gamma}_{f}$ on the boundary $\partial \mathcal{F}_{\bar{\rho}}$ as follows. If we let

$$
\mathbf{v}_{f}:=\mathbf{F}^{T} \mathbf{s}_{f}
$$

then (45) becomes $\mathbf{v}_{f}^{T} \mathbf{D}\left(e^{\boldsymbol{\Gamma}_{f}}\right)=\bar{\rho} \mathbf{s}_{f}^{T}$. From which,

$$
\Gamma_{f}^{(i)}=\log \left(\bar{\rho} s_{f}^{(i)} / v_{f}^{(i)}\right) ; i=1, \cdots, K .
$$

After right-multiplying (45) by $\mathbf{F}$ and using (46), it is clear that $\mathbf{v}_{f}^{T} \mathbf{D}\left(e^{\boldsymbol{\Gamma}_{f}}\right) \mathbf{F}=\bar{\rho} \mathbf{v}_{f}^{T}$, i.e., $\mathbf{v}_{f}$ is a left eigenvector associated with eigenvalue $\bar{\rho}$ of $\mathbf{D}\left(e^{\boldsymbol{\Gamma}_{f}}\right) \mathbf{F}$. Furthermore, it can be shown that $\bar{\rho}=\rho\left(\mathbf{D}\left(e^{\boldsymbol{\Gamma}_{f}}\right) \mathbf{F}\right)=\rho\left(\mathbf{F D}\left(e^{\boldsymbol{\Gamma}_{f}}\right)\right)$ [22, Th. 1.3.20].

Once $s_{f}^{(i)}$ is known, the computation of $\Gamma_{f}^{(i)}$ in (47) requires $v_{f}^{(i)}$ to be found by (46). However, as $\mathbf{F}$ involves a matrix inverse operation [see (42)], it is not yet straightforward to find $v_{f}^{(i)}$ distributively. Using (42), we rewrite (46) as

$$
\begin{aligned}
\mathbf{v}_{f}^{T}= & \mathbf{s}_{f}^{T} \\
& \mathbf{H}_{21} \mathbf{D}\left(e^{\boldsymbol{\Gamma}_{m}^{\min }}\right)\left[\bar{\rho} \mathbf{I}_{M}-\mathbf{H}_{11} \mathbf{D}\left(e^{\boldsymbol{\Gamma}_{m}^{\mathrm{min}}}\right)\right]^{-1} \mathbf{H}_{12} \\
& +\mathbf{s}_{f}^{T} \mathbf{H}_{22},
\end{aligned}
$$

and define $\mathbf{s}_{m} \in \mathbb{R}_{+}^{M}$ such that

$$
\mathbf{s}_{m}^{T}:=\mathbf{s}_{f}^{T} \mathbf{H}_{21} \mathbf{D}\left(e^{\boldsymbol{\Gamma}_{m}^{\mathrm{min}}}\right)\left[\bar{\rho} \mathbf{I}_{M}-\mathbf{H}_{11} \mathbf{D}\left(e^{\boldsymbol{\Gamma}_{m}^{\mathrm{min}}}\right)\right]^{-1} .
$$

Proposition 6: Given an initialization $\mathbf{s}_{m}^{T}[0] \succ \mathbf{0}, \mathbf{s}_{m}^{T}$ can be realized by the following update:

$\mathbf{s}_{m}^{T}[t+1]=\frac{1}{\bar{\rho}} \mathbf{s}_{m}^{T}[t] \mathbf{H}_{11} \mathbf{D}\left(e^{\boldsymbol{\Gamma}_{m}^{\min }}\right)+\frac{1}{\bar{\rho}} \mathbf{s}_{f}^{T} \mathbf{H}_{21} \mathbf{D}\left(e^{\boldsymbol{\Gamma}_{m}^{\mathrm{min}}}\right)$.

Proof: The proof is given in Appendix C.

Notice that the $i$-th component of $\mathbf{s}_{m}[t+1]$ in (50) is actually

$s_{m}^{(i)}[t+1]=\frac{e^{\Gamma_{m}^{\min (i)}}}{\bar{\rho}}\left[\sum_{j=1}^{M} H_{11}^{(j, i)} s_{m}^{(j)}[t]+\sum_{j=1}^{K} H_{21}^{(j, i)} s_{f}^{(j)}\right]$

for $i=1, \cdots, M$. From (1) and upon recalling the partition of $\mathbf{H}$, the update in (51) further amounts to

$$
\begin{array}{r}
s_{m}^{(i)}[t+1]=\frac{e^{\Gamma_{m}^{\min (i)}}}{\bar{\rho}}\left[\sum_{j \in \mathcal{L}_{m} \backslash\{i\}} s_{m}^{(j)}[t]+\sum_{j \in \mathcal{L}_{f}} h_{\sigma_{j}, i} s_{f}^{(j-K)}\right] \\
=\frac{e^{\Gamma_{m}^{\min (i)}}}{\bar{\rho}}\left[\sum_{j \in \mathcal{L}_{m} \backslash\{i\}} s_{m}^{(j)}[t]+\sum_{l \neq \sigma} h_{l, i} \sum_{j, \sigma_{j}=l} s_{f}^{(j-K)}\right](52)
\end{array}
$$

for $i=1, \cdots, M$. Clearly, $s_{m}^{(i)}[t+1]$ consists of the internal component $\sum_{j \in \mathcal{L}_{m} \backslash\{i\}} s_{m}^{(j)}[t]$ due to other MUEs, and the $e x$ ternal component $\sum_{l \neq \sigma} h_{l, i} \sum_{j, \sigma_{j}=l} s_{f}^{(j-K)}$ due to all FUEs (with $\sigma$ denoting the macrocell $\mathrm{BS}$ ).

With $\mathbf{s}_{f} \succ \mathbf{0}$ known and once $\mathbf{s}_{m} \succ \mathbf{0}$ has been determined, $\mathbf{v}_{f}$ can readily be computed. From (48) and (49), $\mathbf{v}_{f}^{T}=\mathbf{s}_{m}^{T} \mathbf{H}_{12}+\mathbf{s}_{f}^{T} \mathbf{H}_{22}$. Then, its component can be found according to

$v_{f}^{(i)}=h_{\sigma, i} \sum_{j \in \mathcal{L}_{m}} s_{m}^{(j)}+\sum_{j \neq i, \sigma_{j}=\sigma_{i}} s_{f}^{(j)}+\sum_{l \neq \sigma_{i}} h_{l, i} \sum_{j, \sigma_{j}=l} s_{f}^{(j)}(53)$

for $i=1, \cdots, K$. It is worth commenting on that the first term of (53) amounts to the effects from all MUEs, whereas the second term from the FUEs within the same femtocell, and the third term from the FUEs in all other femtocells.

\section{B. Distributed Algorithm for Femtocell Utility Maximization and Macrocell SINR Balancing}

The above parametrization $\boldsymbol{\Gamma}_{f}=\boldsymbol{\Gamma}_{f}\left(\mathbf{s}_{f}, \boldsymbol{\Gamma}_{m}^{\min }, \bar{\rho}\right)$ allows us to find all points on $\mathcal{F}_{\bar{\rho}}$. By fixing $\bar{\rho} \in[0,1)$ and upon applying that parametrization, (40) can be solved via an equivalent optimization problem, albeit in the new variable $\mathbf{s}_{f}$. The latter involves finding a direction of $\mathbf{s}_{f}$ that leads $\boldsymbol{\Gamma}_{f}$ and $\mathbf{p}$ to the optimum of the original problem. With $\varepsilon>0$, we propose to update $s_{f}^{(i)}$ as

$$
s_{f}^{(i)}[t+1]:=s_{f}^{(i)}[t]+\varepsilon \Delta s_{f}^{(i)}[t+1],
$$

for $i=1, \cdots, K$, where

$$
\Delta s_{f}^{(i)}[t+1]:=U_{i}^{\prime}\left(\Gamma_{f}^{(i)}\right) /\left(\bar{\rho} q_{f}^{(i)}\right)-s_{f}^{(i)}[t] .
$$

Upon recalling that $\mathbf{s}_{f}$ is a left eigenvector associated with eigenvalue $\bar{\rho}$ of $\mathbf{F D}\left(e^{\boldsymbol{\Gamma}_{f}}\right)$, it can be proven that the update of $\mathbf{s}_{f}$ in (54)-(55) actually represents an ascent direction for $U\left(\mathbf{s}_{f}\right)=\sum_{i \in \mathcal{L}_{f}} U_{i}\left(\Gamma^{(i)}\right)$ [20]. We also note that the update of MUE load $s_{m}^{(i)}$ in (52) is totally different from that of FUE load $s_{f}^{(i)}$ in (54).

We present in Alg. 2 the Femtocell Utility Maximization with Macrocell SINR Balancing (FUM-MSB) algorithm. Again, it is assumed that the channel gains between the downlink and the uplink are identical. Here, $s_{m}^{(i)}[t+1]$ in Step 4 is computed and managed by MUE $i \in \mathcal{L}_{m}$. Specifically, each femtocell BS $l$ is required to broadcast $\sum_{j, \sigma_{j}=l} s_{f}^{(j)}$ at a constant power. This allows MUE $i$ to also measure all channel gains $h_{l, i}=h_{i, l}$ required for the calculation of $\sum_{l \neq \sigma_{i}} h_{l, i} \sum_{j, \sigma_{j}=l} s_{f}^{(j)}$. On the other hand, macrocell BS communicates the quantity $\sum_{j \in \mathcal{L}_{m}} s_{m}^{(j)}[t]$ to all MUEs, which then permits MUE $i$ to easily compute $\sum_{j \in \mathcal{L}_{m} \backslash\{i\}} s_{m}^{(j)}[t]=$ $\sum_{j \in \mathcal{L}_{m}} s_{m}^{(j)}[t]-s_{m}^{(i)}[t]$. Finally, MUE $i$ reports the resulting $s_{m}^{(i)}[t+1]$ back to macrocell BS for the computation of $\mathbf{s}_{m}$ in the next iteration. Note that each femtocell BS $l$ only needs to broadcast $\sum_{j, \sigma_{j}=l} s_{f}^{(j)}$ once. As well, $\sum_{j \in \mathcal{L}_{m}} s_{m}^{(j)}[t]$ and $s_{m}^{(i)}[t+1]$ can be locally exchanged between macrocell BS and MUE $i$ over the control channel of link $i$.

The computation of $v_{f}^{(j)}$ in Step 7 can also be done by FUE $j$. Once $\mathbf{s}_{m}$ has been determined (i.e., its update 


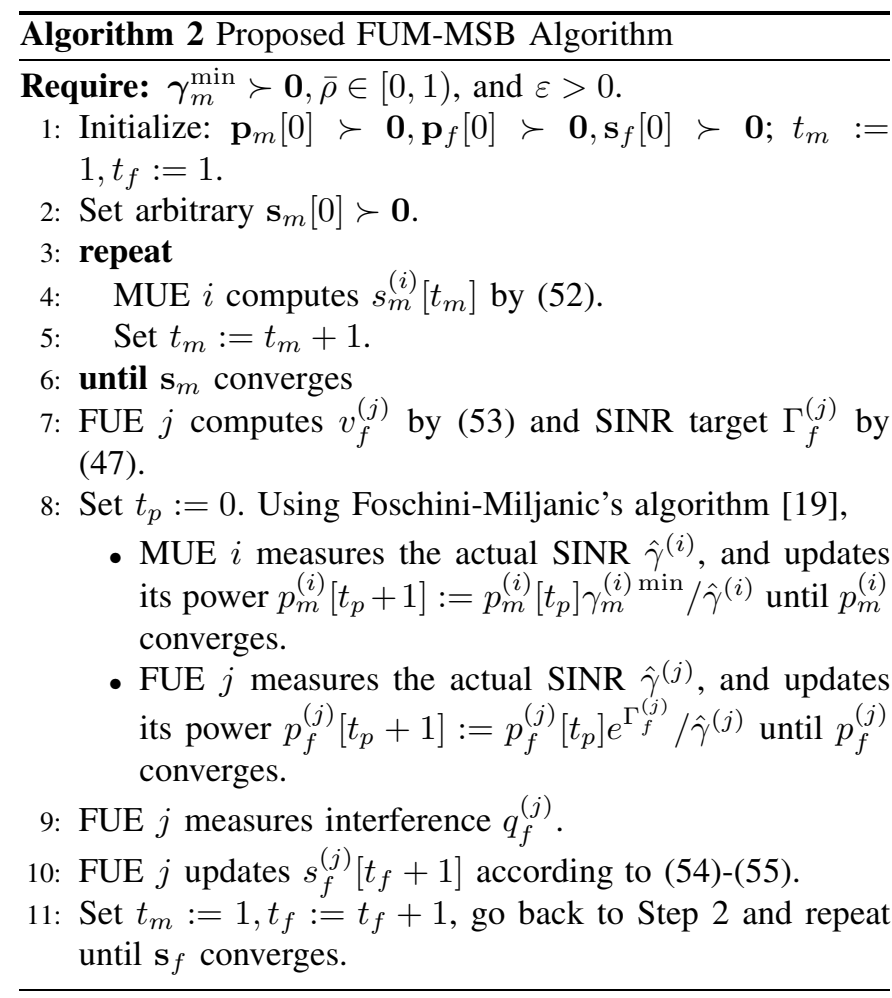

(52) has converged), macrocell BS broadcasts the quantity $\sum_{i \in \mathcal{L}_{m}} s_{m}^{(i)}$, again at a constant power. Recall that all summations $\sum_{i, \sigma_{i}=l} s_{f}^{(i)}$ have already been received at FUE $j$ from all femtocell BSs $l$ (including the one that serves FUE $j$ ). Together with the assumption of symmetric downlink-uplink channel gains, $v_{f}^{(j)}$ can thus be computed according to (53). As well, the update of $s_{f}^{(j)}$ in Step 10 can be accomplished in a completely distributed manner by FUE $j$ with only local information required. Over its own control channel, FUE $j$ then reports the new value of $s_{f}^{(j)}$ to its servicing femtocell $\mathrm{BS} \sigma_{j}$, to be used in the next iteration.

Theorem 2: For a sufficiently small $\varepsilon>0$ and as $\bar{\rho} \rightarrow 1$, the proposed FUM-MSB algorithm converges to the globally optimal solution of (40).

Proof: The idea of this proof is similar to that of Prop. 2. Note that the constraints $\Gamma^{(i)} \geq \Gamma^{(i) \min }, \forall i \in \mathcal{L}_{m}$ in (40) are already satisfied with equality as we are operating on $\partial \mathcal{F}_{\bar{\rho}}$. With multiplier $\mu \geq 0$, the Lagrangian of problem (40) is defined as

$$
\mathcal{L}(\boldsymbol{\Gamma}, \mu):=\sum_{i \in \mathcal{L}_{f}} U_{i}\left(\Gamma^{(i)}\right)-\mu\left[\rho\left(\mathbf{H D}\left(e^{\boldsymbol{\Gamma}}\right)\right)-\bar{\rho}\right] .
$$

The KKT condition of (40) is simply $\nabla \mathcal{L}(\boldsymbol{\Gamma}, \mu)=0$, which can be shown equivalent to

$$
U_{i}^{\prime}\left(\Gamma_{f}^{(i)}\right)=\mu v_{f}^{(i)} \tilde{q}_{f}^{(i)} ; i=1, \cdots, K,
$$

where $\mathbf{v}_{f}=\left[v_{f}^{(i)}\right]$ is the left eigenvector of $\mathbf{D}\left(e^{\boldsymbol{\Gamma}_{f}}\right) \mathbf{F}$ and $\tilde{\mathbf{q}}_{f}=\left[\tilde{q}_{f}^{(i)}\right]$ the right eigenvector of $\mathbf{F D}\left(e^{\boldsymbol{\Gamma}_{f}}\right)$, both associated with eigenvalue $\bar{\rho}$.

At the point of convergence $\mathbf{s}_{f}^{*}$, we have that $\Delta \mathbf{s}_{f}^{*}=0$. It follows from (55) that

$$
U_{i}^{\prime}\left(\Gamma_{f}^{(i) *}\right)=v_{f}^{(i) *} q_{f}^{(i) *} ; i=1, \cdots, K .
$$

Manipulating (5)-(6) and using the matrix/vector partitions specified at the beginning of Sec. IV-A give $\mathbf{q}_{f}=$ $\mathbf{F D}\left(e^{\boldsymbol{\Gamma}_{f}}\right) \mathbf{q}_{f}+\tilde{\boldsymbol{\varphi}}_{f}$ where $\tilde{\boldsymbol{\varphi}}_{f}=\mathbf{H}_{21} \mathbf{D}\left(e^{\boldsymbol{\Gamma}_{m}^{\mathrm{min}}}\right) \boldsymbol{\varphi}_{m}+\boldsymbol{\varphi}_{f}$. By the similar argument used in the proof of Prop. 2, it can be shown that

$$
q_{f}^{(i) *} \rightarrow \tilde{q}_{f}^{(i)} /\left[(1-\bar{\rho}) \mathbf{s}_{f}^{* T} \tilde{\varphi}_{f}\right]
$$

as $\bar{\rho} \rightarrow 1$. Therefore, (58) is exactly (57) for $\boldsymbol{\Gamma}_{f}=\boldsymbol{\Gamma}_{f}^{*}$ and $\mu=1 /\left[(1-\bar{\rho}) \mathbf{s}_{f}^{* T} \tilde{\varphi}_{f}\right]$ in the limit $\bar{\rho} \rightarrow 1$. Since (40) is a convex optimization problem, any point that satisfies the KKT conditions is also its globally optimal solution.

With $\mathbf{s}_{f}^{*}$ known and upon recalling that $\mathbf{v}_{f}^{*}=\mathbf{F}^{T} \mathbf{s}_{f}^{*}$, the optimal SINR assignment $\boldsymbol{\Gamma}_{f}^{*}$ of all FUEs is determined according to (47). Also recollect that the optimal macrocell SINR is indeed $\Gamma_{m}^{*}=\Gamma_{m}^{\min }=\Gamma^{\text {min }} \in \mathbb{R}^{M}$. By Foschini-Miljanic's algorithm [19], the power allocation $\mathbf{p}^{*}$ that achieves these SINR targets can be found. Together with $\boldsymbol{\Gamma}^{*}=\left[\boldsymbol{\Gamma}^{\mathrm{min}} ; \boldsymbol{\Gamma}_{f}^{*}\right], \mathbf{p}^{*}$ gives the global optimum of problem (40).

\section{Advantages of FUM-MSB Algorithm over JUM-QoS Algo- rithm}

Although both proposed schemes can be used to solve the same problem (40), FUM-MSB algorithm outperforms JUMQoS algorithm in this specific case. Compared with JUM-QoS, FUM-MSB solution converges more quickly to the optimal points. This is because the latter operates independently of the total number of MUEs $M$, and its search space for Pareto-optimal SINR is simply confined to $\mathbb{R}^{K}$. In practical networks with a large number of MUEs, FUM-MSB algorithm is therefore more scalable. Moreover, this solution offers a substantial reduction in computational complexity. Recall that JUM-QoS algorithm is based on the update of the penalty factor $a$ in another time scale, and hence its actual performance can be sensitive to the value of $a$. On the contrary, penalty method is not needed at all in the FUM-MSB scheme. Further, this specific algorithm does not need to search for a feasible starting point (similar to that in (30)-(31)), which involves some message exchange among all macrocell and femtocell BSs. Given that only limited backhaul network capacity is available for femtocells, this kind of message passing can be a performance bottleneck in certain scenarios. Note also that the scaling of step size $\delta$ in Step 7 of the JUM-QoS algorithm might lead to the ripple effect around the optimum, as one tries to push $\Gamma$ into the strict interior of the feasible set. This instability issue does not happen to the FUM-MSB solution.

\section{NUMERICAL EXAMPLES}

We present numerical results to illustrate the performance of the two proposed algorithms JUM-QoS and FUM-MSB. The network setting and user placement in our examples are shown in Fig. 1, where MUEs and FUEs are randomly deployed inside circles of radii of $1000 \mathrm{~m}$ and $50 \mathrm{~m}$, respectively. In particular, we assume there are $M=10$ MUEs, whereas $K=20$ FUEs are equally divided among 4 femtocells (i.e., 5 FUEs per femtocell). The uplink case is considered in all 


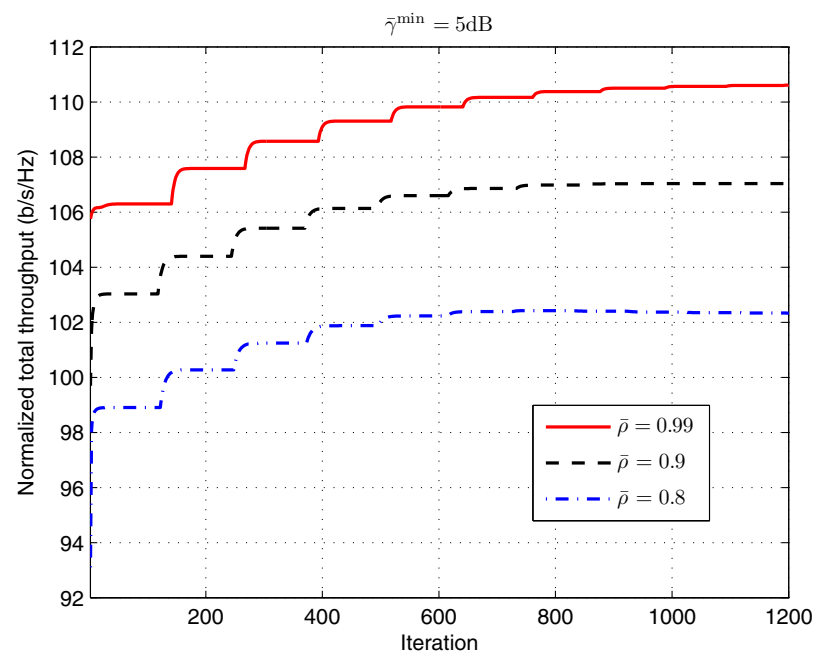

Fig. 3. Convergence process of the JUM-QoS algorithm.

simulations. The absolute channel gain from the transmitter of user $j$ to $\mathrm{BS} \sigma_{i}$ that serves user $i$ is calculated as

$$
\bar{h}_{\sigma_{i}, j}= \begin{cases}d_{\sigma_{i} j}^{-3}, & \text { if } \sigma_{j}=\sigma_{i}, \\ d_{\sigma_{i}, j}^{-3} /\left(10^{\kappa / 10}\right), & \text { if } \sigma_{j} \neq \sigma_{i},\end{cases}
$$

where $d_{\sigma_{i}, j}$ is their corresponding geographical distance, and $\kappa=10 \mathrm{~dB}$ is used to represent the extra cross-cell signal loss due to penetration through walls (as FUEs are typically deployed indoors).

For simplicity, we consider unit bandwidth. The throughput, normalized over the total bandwidth, is thus expressed in terms of $\mathrm{b} / \mathrm{s} / \mathrm{Hz}$. Gaussian noise power is taken as $\varphi^{(i)}=10^{-6}, \forall i \in \mathcal{L}$. Normalized minimum SINRs $\gamma_{m}^{\min }=\left[\gamma^{(1) \min }, \cdots, \gamma^{(M) \min }\right]^{T}$, are assumed equal for all MUEs, i.e., $\gamma^{(i) \min }=\gamma^{\text {min }}, \forall i \in \mathcal{L}_{m}$, chosen such that $\rho\left(\mathbf{H D}\left(\left[\boldsymbol{\gamma}_{m}^{\min } ; \mathbf{0}_{K}\right]\right)\right) \leq \bar{\rho}<1$. Because all the results obtained in the previous sections are applied to the normalized SINR $\gamma^{(i)}, \forall i \in \mathcal{L}$, the actual attained SINR in the numerical examples must be recovered according to $\bar{\gamma}^{(i)}=G \gamma^{(i)}, \forall i \in$ $\mathcal{L}$, where $G$ is the processing gain. While it is possible to select other values of $G$, we choose $G=32$ for this particular network realization so that the actual minimum SINR $\bar{\gamma}^{\mathrm{min}}=G \gamma^{\mathrm{min}}$ is within a practical range (i.e., from $5 \mathrm{~dB}$ to almost $8.5 \mathrm{~dB}$ ). Unless stated otherwise, 3 -fair utility function is used, i.e., $\alpha=3$ in (11). We set the error tolerance for the convergence of the proposed schemes and Foschini-Miljanic's algorithm as $\epsilon=10^{-4}$ and $\epsilon_{p}=10^{-10}$, respectively. For JUM-QoS algorithm, $a=2, k=2, \delta=0.1, b=0.8$ are assumed, whereas for FUM-MSB algorithm $\varepsilon=0.1$.

Fig. 3 demonstrates the convergence of JUM-QoS algorithm for $w_{m}=w_{f}=0.5$. At each stage of the proposed log-barrier penalization (which corresponds to a given penalty factor $a$ ), the algorithm quickly converges in some tens of iterations, and an improvement in the normalized total throughput is observed. After several updates of $a$, the final convergence is realized. As $\bar{\rho}$ tends to 1 , the total sum rates of all MUEs and FUEs increase. This is because the feasible SINR region becomes larger as $\bar{\rho}$ grows, meaning that more capacity is available for use. It should also be noted that JUM-QoS is expected to approach the global optimum in the limit $\bar{\rho} \rightarrow 1$

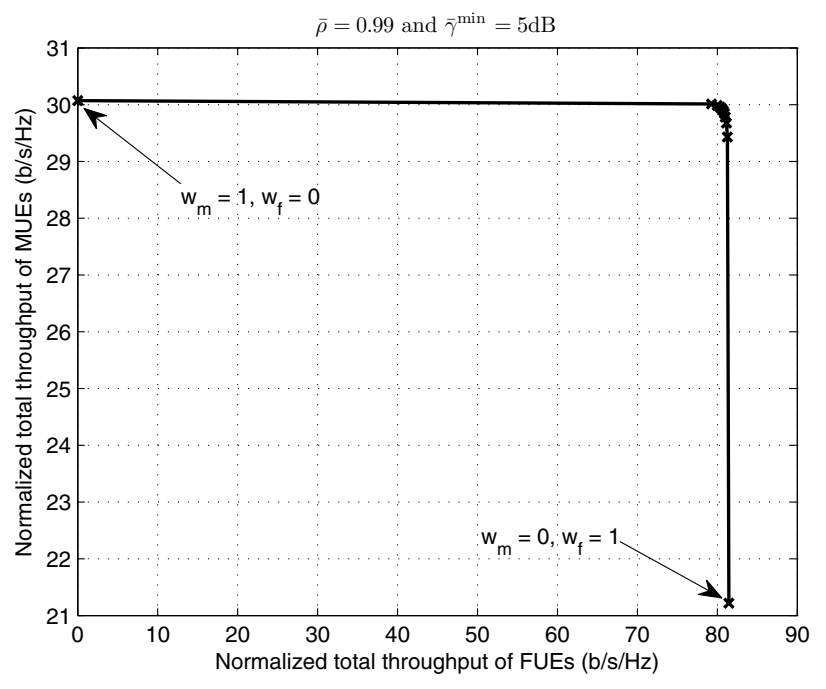

Fig. 4. Throughput tradeoff between macrocell and femtocell networks by the JUM-QoS algorithm.

[see Prop. 2 and Th. 1].

On the other hand, the results presented in Table I can be interpreted as follows. Without any MUEs' prescribed minimum SINR $\bar{\gamma}_{m}^{\min }$, the optimal SINR assignment for all MUEs that maximizes the joint utilities of both macrocell and femtocell networks can be denoted as $\check{\gamma}_{m}^{*}$, whose entries range from $8.3637 \mathrm{~dB}$ to $8.4331 \mathrm{~dB}$. As such, including any SINR $\bar{\gamma}_{m}^{\min } \preceq \check{\gamma}_{m}^{*}$ in the constraint set does not change this final solution. While different network configurations correspond to different specific values of $\check{\gamma}_{m}^{*}$, this solution $\check{\gamma}_{m}^{*}$ will no longer be feasible for any constraint $\bar{\gamma}_{m}^{\min } \succ \check{\gamma}_{m}^{*}$. Remarkably, the proposed JUM-QoS algorithm always guarantees that the resulting SINRs of all MUEs be actually greater than $\bar{\gamma}_{m}^{\min }$ in that case, as evidenced in the last three columns of Table I. Furthermore, it is noteworthy that a small variation in $\bar{\gamma}_{m}^{\min }$ in this range of SINR may significantly reduce the remaining network capacity available for the FUEs. From Table I, as the prioritized MUEs demand for a slight increase of $0.025 \mathrm{~dB}$ in $\bar{\gamma}_{m}^{\min }$, the total throughput of all femtocells is decreased by half, dropping from almost $75 \mathrm{~b} / \mathrm{s} / \mathrm{Hz}$ to about $37.5 \mathrm{~b} / \mathrm{s} / \mathrm{Hz}$.

To flexibly share the radio resources among MUEs and FUEs, the general JUM-QoS algorithm can designate the importance toward either macrocell or femtocell network by varying $w_{m}$ and $w_{f}$. In Fig. 4, the achieved throughput of both networks is displayed for $w_{m}=0: 0.1: 1$ and $w_{f}=1-w_{m}$. Clearly, by changing from $w_{m}=1$ to $w_{m}=0$, i.e., MUEs only require to have their minimum QoS maintained rather than their utility solely optimized, the throughput improvement in the femtocell network is ninefold the amount of rate loss in the macrocell. Such a pronounced gain can be explained by noting that FUEs are located in close proximity to their corresponding BSs, and thus are able to achieve potentially much higher data rates compared to MUEs.

In order to compare its performance with that of the specific FUM-MSB algorithm, we set $w_{m}=0, w_{f}=1$ in the general JUM-QoS algorithm. With $\bar{\rho}=0.99$ and $\bar{\gamma}_{m}^{\min }=8.4 \mathrm{~dB}$, this general algorithm takes almost $10^{5}$ iterations to reach the final optimal solution (see Fig. 5(a)). The main reason 
TABLE I

PERFormanCE OF JUM-QOS ALGORITHM $(\bar{\rho}=0.99)$.

\begin{tabular}{|c||c|c|c|c|c|c|}
\hline $\bar{\gamma}^{\text {min }}(\mathrm{dB})$ & 6 & 7 & 8 & 8.4 & 8.45 & 8.475 \\
\hline$\rho\left(\mathbf{H D}\left(\left[\boldsymbol{\gamma}_{m}^{\min }, \mathbf{0}_{K}\right]\right)\right)$ & 0.5598 & 0.7048 & 0.8873 & 0.9729 & 0.9842 & 0.9898 \\
\hline $\max \left\{\bar{\gamma}_{m}\right\}(\mathrm{dB})$ & 8.4331 & 8.4331 & 8.4331 & 8.4895 & 8.4688 & 8.4754 \\
\hline $\min \left\{\bar{\gamma}_{m}\right\}(\mathrm{dB})$ & 8.3637 & 8.3637 & 8.3637 & $\mathbf{8 . 4 0 0 0}$ & $\mathbf{8 . 4 5 0 0}$ & $\mathbf{8 . 4 7 5 0}$ \\
\hline Total normalized throughput of all MUEs $(\mathrm{b} / \mathrm{s} / \mathrm{Hz})$ & 29.8930 & 29.8930 & 29.8930 & 29.8955 & 30.0110 & 30.0702 \\
\hline Total normalized throughput of all FUEs $(\mathrm{b} / \mathrm{s} / \mathrm{Hz})$ & 80.7477 & 80.7477 & 80.7477 & 80.5960 & 74.7508 & 37.5895 \\
\hline
\end{tabular}

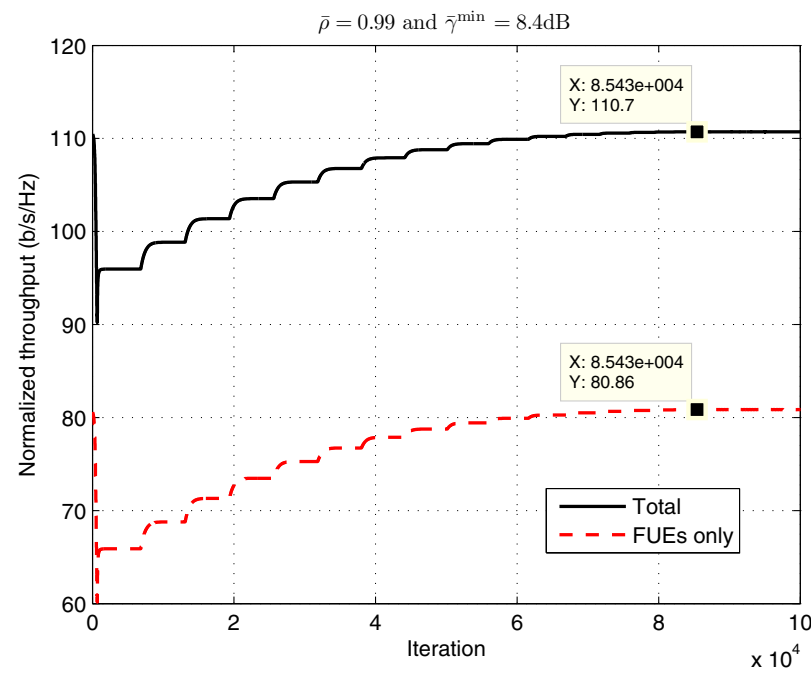

(a) JUM-QoS

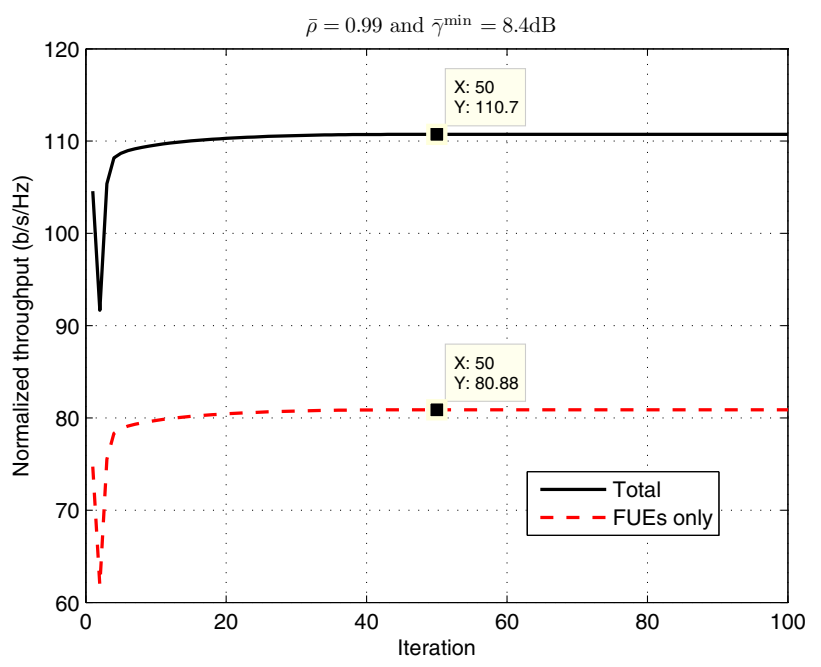

(b) FUM-MSB

Fig. 5. Performance comparison of JUM-QoS and FUM-MSB algorithms.

for such a long converging time is that it takes the logbarrier penalty method quite a lot of efforts to push the MUEs' SINRs to be so close to the boundary of the feasible SINR region, i.e., to achieve $\bar{\gamma}_{m}^{\min }$. Even so, since JUM-QoS algorithm operates strictly inside the feasible region, macrocell SINR targets can never get exactly equal to $8.4 \mathrm{~dB}$. On the contrary, FUM-MSB algorithm settles very quickly to the global optimum in as few as 10 iterations (see Fig. 5(b)), with the exact SINR $8.4 \mathrm{~dB}$ obtained for all the MUEs. The latter fact also explains why the total femtocell throughput given

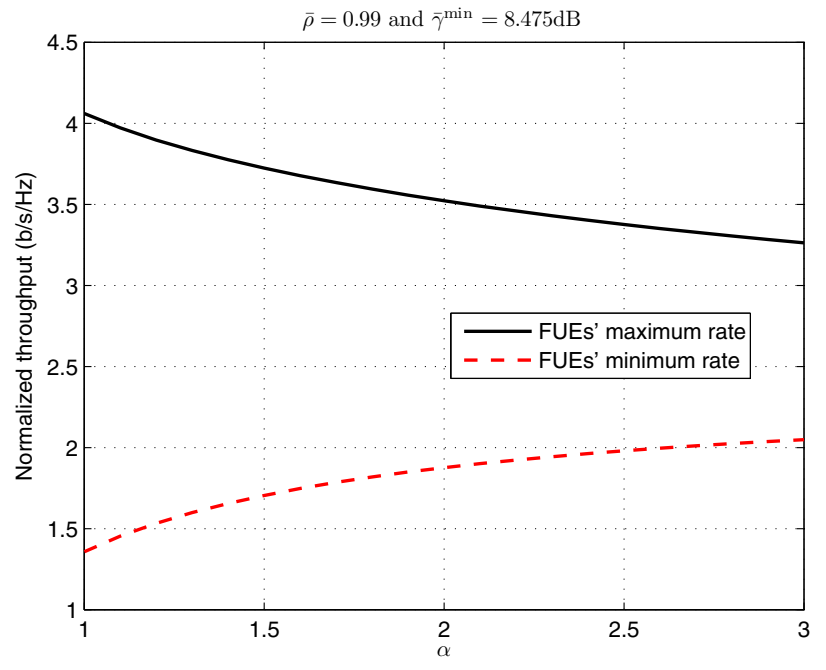

Fig. 6. Fairness achieved by the use of different utility functions in the FUM-MSB algorithm.

by this algorithm is somewhat greater than that by the JUMQoS counterpart. Moreover, computational results suggest that FUM-MSB algorithm does not experience any ripple effect that occurs to the JUM-QoS scheme around the optimum point.

The issue of fairly utilizing the available radio resources can be effectively resolved by regulating $\alpha$ in the utility function. Fig. 6 shows the minimum and maximum throughput of all the femtocells, given by the FUM-MSB solution for different values of $\alpha$. Apparently, as $\alpha$ increases, the FUE whose data rate is the highest (likely due to its advantageous link condition) sees a decline in its throughput, whereas the FUE with the lowest throughput has its data rate gradually enhanced. For a large value of $\alpha$, the FUEs' minimum throughput is further expected to reach a plateau, meaning that max-min fairness is realized at that point.

\section{CONCLUSION}

In heterogeneous networks, the critical requirement of protecting the QoS of all MUEs poses a key challenge that hinders the application of any available interference management solutions. This paper attempts to overcome that major difficulty, for which the load-spillage approach can be successfully adapted. Specifically, two distributed Pareto-optimal power control schemes have been proposed. The general algorithm JUM-QoS jointly maximizes the total utility of both macrocell and femtocell networks while robustly protecting the prescribed minimum SINRs of MUEs at all times. In 
the special case where the macrocell network only needs to be assured with some certain minimum QoS, the second algorithm FUM-MSB has also been developed to offer several practical advantages over its general counterpart JUM-QoS. It has been shown that the two devised solutions converge to their respective global optima. The potentials of the proposed approaches have been demonstrated by numerical examples.

\section{APPENDIX A}

\section{DERIVATION OF GRADIENT OF SPECTRAL RADIUS}

In this appendix, we provide a complete derivation of the gradient of the spectral radius of $\mathbf{D}\left(e^{\boldsymbol{\Gamma}}\right) \mathbf{H}$ and $\mathbf{H D}\left(e^{\boldsymbol{\Gamma}}\right)$. By [22, Th. 1.3.20], $\mathbf{D}\left(e^{\boldsymbol{\Gamma}}\right) \mathbf{H}$ and $\mathbf{H D}\left(e^{\boldsymbol{\Gamma}}\right)$ indeed have the same set of eigenvalues, counting multiplicity. This implies that they have the same eigenvalue of maximum modulus. Denote $\bar{\rho}:=$ $\rho\left(\mathbf{D}\left(e^{\boldsymbol{\Gamma}}\right) \mathbf{H}\right)=\rho\left(\mathbf{H D}\left(e^{\boldsymbol{\Gamma}}\right)\right)$. Also, let s and $\tilde{\mathbf{q}}$ respectively be the left and right eigenvectors associated with $\bar{\rho}$ of $\mathbf{H D}\left(e^{\boldsymbol{\Gamma}}\right)$, normalized such that $\mathbf{s}^{T} \tilde{\mathbf{q}}=1$. Then,

$$
\begin{aligned}
\mathbf{s}^{T} \mathbf{H D}\left(e^{\boldsymbol{\Gamma}}\right) & =\bar{\rho} \mathbf{s}^{T}, \\
\mathbf{H D}\left(e^{\boldsymbol{\Gamma}}\right) \tilde{\mathbf{q}} & =\bar{\rho} \tilde{\mathbf{q}} .
\end{aligned}
$$

Define $\mathbf{v}:=\mathbf{H}^{T} \mathbf{s}$. Upon right-multiplying both sides of (61) by $\mathbf{H}$, it is clear that $\mathbf{v}^{T} \mathbf{D}\left(e^{\boldsymbol{\Gamma}}\right) \mathbf{H}=\bar{\rho} \mathbf{v}^{T}$, i.e., $\mathbf{v}$ is a left eigenvector associated with $\bar{\rho}$ of $\mathbf{D}\left(e^{\boldsymbol{\Gamma}}\right) \mathbf{H}$. Now, taking the gradient of (62) with respect to $\Gamma$ yields

$$
\mathbf{H}\left(\nabla \mathbf{D}\left(e^{\boldsymbol{\Gamma}}\right)\right) \tilde{\mathbf{q}}+\mathbf{H D}\left(e^{\boldsymbol{\Gamma}}\right) \nabla \tilde{\mathbf{q}}=(\nabla \bar{\rho}) \tilde{\mathbf{q}}+\bar{\rho} \nabla \tilde{\mathbf{q}} .
$$

After left-multiplying both sides of (63) with $\mathbf{s}^{T}$ and using (61), we arrive at

$$
\mathbf{s}^{T} \mathbf{H}\left(\nabla \mathbf{D}\left(e^{\boldsymbol{\Gamma}}\right)\right) \tilde{\mathbf{q}}=(\nabla \bar{\rho}) \mathbf{s}^{T} \tilde{\mathbf{q}} .
$$

Recall that $\mathbf{v}=\mathbf{H}^{T} \mathbf{s}$ and $\mathbf{s}^{T} \tilde{\mathbf{q}}=1$, then (64) can be shown equivalent to

$$
\partial \bar{\rho} / \partial \Gamma^{(i)}=v^{(i)} \tilde{q}^{(i)} e^{\Gamma^{(i)}} ; i=1 \cdots, M+K .
$$

\section{APPENDIX B}

\section{PROOF OF PROPOSITION 4}

Let $\mathbf{s}$ be the left eigenvector of $\operatorname{HD}\left(e^{\boldsymbol{\Gamma}}\right)$ with associated eigenvalue $\bar{\rho}=\rho\left(\mathbf{H D}\left(e^{\boldsymbol{\Gamma}}\right)\right) \in[0,1)$. Therefore, $\mathbf{s}^{T} \mathbf{H D}\left(e^{\boldsymbol{\Gamma}}\right)=\bar{\rho} \mathbf{s}^{T}$, which can also be explicitly expressed as

$$
\left[\mathbf{s}_{m}^{T}, \mathbf{s}_{f}^{T}\right]\left[\begin{array}{ll}
\mathbf{H}_{11} & \mathbf{H}_{12} \\
\mathbf{H}_{21} & \mathbf{H}_{22}
\end{array}\right] \mathbf{D}\left(e^{\boldsymbol{\Gamma}_{m}^{\min }}, e^{\boldsymbol{\Gamma}_{f}}\right)=\bar{\rho}\left[\mathbf{s}_{m}^{T}, \mathbf{s}_{f}^{T}\right],
$$

where $\mathbf{s}_{m}^{T}=\left[s_{1}, \cdots, s_{M}\right]$ and $\mathbf{s}_{f}^{T}=\left[s_{M+1}, \cdots, s_{M+K}\right]$.

By $[22$, Th. 5.6.9 \& Cor. 5.6.16], the assumption $\rho\left(\mathbf{H}_{11} \mathbf{D}\left(e^{\boldsymbol{\Gamma}_{m}^{\min }}\right)\right)<\bar{\rho}$ ensures that $\left[\bar{\rho} \mathbf{I}_{M}-\right.$ $\left.\mathbf{H}_{11} \mathbf{D}\left(e^{\boldsymbol{\Gamma}_{m}^{\min }}\right)\right]^{-1}=(1 / \bar{\rho}) \sum_{k=0}^{\infty}\left[(1 / \bar{\rho}) \mathbf{H}_{11} \mathbf{D}\left(e^{\boldsymbol{\Gamma}_{m}^{\min }}\right)\right]^{k}$ exists and is positive componentwise. After some algebraic manipulations, one arrives at

$$
\begin{aligned}
\mathbf{s}_{f}^{T}\{ & \mathbf{H}_{21} \mathbf{D}\left(e^{\boldsymbol{\Gamma}_{m}^{\mathrm{min}}}\right)\left[\bar{\rho} \mathbf{I}_{M}\right. \\
& \left.\left.\quad-\mathbf{H}_{11} \mathbf{D}\left(e^{\boldsymbol{\Gamma}_{m}^{\mathrm{min}}}\right)\right]^{-1} \mathbf{H}_{12}+\mathbf{H}_{22}\right\} \mathbf{D}\left(e^{\boldsymbol{\Gamma}_{f}}\right)=\bar{\rho} \mathbf{s}_{f}^{T} .
\end{aligned}
$$

With $\mathbf{F}$ defined in (42), the left-hand side of (67) is actually $\mathbf{s}_{f}^{T} \mathbf{F D}\left(e^{\boldsymbol{\Gamma}_{f}}\right)$. This implies that $\bar{\rho}$ is also an eigenvalue of
$\mathbf{F D}\left(e^{\boldsymbol{\Gamma}_{f}}\right)$. Since $\mathbf{H D}\left(e^{\boldsymbol{\Gamma}}\right)$ is primitive, one must have $\mathbf{s} \succ \mathbf{0}$, from which $\mathbf{s}_{f} \succ \mathbf{0}$. Apparently, $\mathbf{F}$ is a positive matrix; hence, so is $\mathbf{F D}\left(e^{\boldsymbol{\Gamma}_{f}}\right)$. By Perron's theorem [22, Th. 8.2.11], $\bar{\rho}$ is the unique eigenvalue of maximum modulus of $\mathbf{F D}\left(e^{\boldsymbol{\Gamma}_{f}}\right)$.

\section{APPENDIX C \\ PROOF OF PROPOSITION 6}

By recursively substituting into (50) and taking the limit $t \rightarrow \infty$, we have that

$$
\begin{aligned}
\lim _{t \rightarrow \infty} \mathbf{s}_{m}^{T}[t]=\mathbf{s}_{m}^{T}[0] \lim _{t \rightarrow \infty}\left[\frac{1}{\bar{\rho}} \mathbf{H}_{11} \mathbf{D}\left(e^{\boldsymbol{\Gamma}_{m}^{\mathrm{min}}}\right)\right]^{t} \\
\quad+\frac{1}{\bar{\rho}} \mathbf{s}_{f}^{T} \mathbf{H}_{21} \mathbf{D}\left(e^{\boldsymbol{\Gamma}_{m}^{\mathrm{min}}}\right) \lim _{t \rightarrow \infty}\left\{\sum_{i=0}^{t-1}\left[\frac{1}{\bar{\rho}} \mathbf{H}_{11} \mathbf{D}\left(e^{\boldsymbol{\Gamma}_{m}^{\mathrm{min}}}\right)\right]^{i}\right\}
\end{aligned}
$$

Recall that if $\rho\left(\mathbf{H}_{11} \mathbf{D}\left(e^{\boldsymbol{\Gamma}_{m}^{\mathrm{min}}}\right)\right)<\bar{\rho}<1$, then it is clear that $\lim _{t \rightarrow \infty}\left[(1 / \bar{\rho}) \mathbf{H}_{11} \mathbf{D}\left(e^{\left.\boldsymbol{\Gamma}_{m}^{\text {min }}\right)}\right]^{t}=0\right.$. By [22, Th. 5.6.9 \& Cor. 5.6.16], $\left[\mathbf{I}_{M}-(1 / \bar{\rho}) \mathbf{H}_{11} \mathbf{D}\left(e^{\boldsymbol{\Gamma}_{m}^{\text {min }}}\right)\right]^{-1}=$ $\sum_{t=0}^{\infty}\left[(1 / \bar{\rho}) \mathbf{H}_{11} \mathbf{D}\left(e^{\boldsymbol{\Gamma}_{m}^{\mathrm{min}}}\right)\right]^{t}$ exists and is positive componentwise. As such, (68) becomes

$$
\begin{gathered}
\lim _{t \rightarrow \infty} \mathbf{s}_{m}^{T}[t]=\frac{1}{\bar{\rho}} \mathbf{s}_{f}^{T} \mathbf{H}_{21} \mathbf{D}\left(e^{\boldsymbol{\Gamma}_{m}^{\min }}\right)\left\{\sum_{i=0}^{\infty}\left[\frac{1}{\bar{\rho}} \mathbf{H}_{11} \mathbf{D}\left(e^{\boldsymbol{\Gamma}_{m}^{\mathrm{min}}}\right)\right]^{i}\right\} \\
=\frac{1}{\bar{\rho}} \mathbf{s}_{f}^{T} \mathbf{H}_{21} \mathbf{D}\left(e^{\boldsymbol{\Gamma}_{m}^{\mathrm{min}}}\right)\left[\mathbf{I}_{M}-\frac{1}{\bar{\rho}} \mathbf{H}_{11} \mathbf{D}\left(e^{\boldsymbol{\Gamma}_{m}^{\mathrm{min}}}\right)\right]^{-1},(69)
\end{gathered}
$$

which is equivalent to (49).

\section{ACKNOWLEDGEMENT}

The authors are thankful to the anonymous reviewers, whose comments have helped improve the presentation of this paper.

\section{REFERENCES}

[1] H. Claussen, L. T. W. Ho, and L. G. Samuel, "An overview of the femtocell concept," Bell Labs. Tech. J., vol. 3, no. 1, pp. 221-245, May 2008.

[2] V. Chandrasekhar, J. G. Andrews, and A. Gatherer, "Femtocell networks: a survey," IEEE Commun. Mag., vol. 46, no. 9, pp. 59-67, Sep. 2008.

[3] C. Patel, M. Yavuz, and S. Nanda, "Femtocells [industry perspectives]," IEEE Wireless Commun., vol. 17, no. 5, pp. 6-7, Oct. 2010.

[4] G. d. 1. Roche, A. Valcarce, D. Lopez-Perez, and J. Zhang, "Access control mechanisms for femtocells," IEEE Commun. Mag., vol. 48, no. 1, pp. 33-39, Jan. 2010.

[5] S. Kishore, L. J. Greenstein, H. V. Poor, and S. C. Schwartz, "Uplink user capacity in a CDMA system with hotspot microcells: effects of finite transmit power and dispersion," IEEE Trans. Wireless Commun., vol. 5, no. 2, pp. 417-426, Feb. 2006.

[6] H. Ji and C.-Y. Huang, "Non-cooperative uplink power control in cellular radio systems," Wireless Netw., vol. 4, no. 3, pp. 233-240, 1998.

[7] Z. Han and K. J. R. Liu, "Noncooperative power-control game and throughput game over wireless networks," IEEE Trans. Commun., vol. 53, no. 10, pp. 1625-1629, Oct. 2005.

[8] E. J. Hong, S. Y. Yun, and D.-H. Cho, "Decentralized power control scheme in femtocell networks: a game theoretic approach," in Proc. 2009 IEEE Int. Symp. on Personal, Indoor and Mobile Radio Commun., pp. $415-419$.

[9] L. Giupponi and C. Ibars, "Distributed interference control in OFDMAbased femtocells," in Proc. 2010 IEEE Int. Symp. on Personal, Indoor and Mobile Radio Commun., pp. 1201-1206.

[10] S. Guruacharya, D. Niyato, E. Hossain, and D. I. Kim, "Hierarchical competition in femtocell-based cellular networks," in Proc. 2010 IEEE Global Commun. Conf., pp. 1-5.

[11] C.-H. Ko and H.-Y. Wei, "On-demand resource-sharing mechanism design in two-tier OFDMA femtocell networks," IEEE Trans. Veh. Technol., vol. 60, no. 3, pp. 1059-1071, Mar. 2011. 
[12] D. T. Ngo, L. B. Le, T. Le-Ngoc, E. Hossain, and D. I. Kim, "Distributed interference management in femtocell networks," IEEE Trans. Wireless Commun., vol. 11, no. 3, pp. 979-989, Mar. 2012.

[13] C. U. Saraydar, N. B. Mandayam, and D. J. Goodman, "Pricing and power control in a multicell wireless data network," IEEE J. Sel. Areas Commun., vol. 19, no. 10, pp. 1883-1892, Oct. 2001.

[14] _ "Efficient power control via pricing in wireless data networks," IEEE Trans. Commun., vol. 50, no. 2, pp. 291-303, Feb. 2002.

[15] M. Xiao, N. B. Shroff, and E. K. P. Chong, "A utility-based power control scheme in wireless cellular systems," IEEE/ACM Trans. Netw., vol. 11, no. 2, pp. 210-221, Apr. 2003.

[16] S. Koskie and Z. Gajic, "A Nash game algorithm for SIR-based power control in 3G wireless CDMA networks," IEEE/ACM Trans. Netw., vol. 13, no. 5, pp. 1017-1026, Oct. 2005.

[17] V. Chandrasekhar, J. G. Andrews, T. Muharemovic, and Z. Shen, "Power control in two-tier femtocell networks," IEEE Trans. Wireless Commun., vol. 8, no. 8, pp. 4316-4328, Aug. 2009.

[18] M. Rasti, A. R. Sharafat, and B. Seyfe, "Pareto-efficient and goal-driven power control in wireless networks: a game-theoretic approach with a novel pricing scheme," IEEE/ACM Trans. Netw., vol. 17, no. 2, pp. 556569, Apr. 2009.

[19] G. J. Foschini and Z. Miljanic, "A simple distributed autonomous power control algorithm and its convergence," IEEE Trans. Veh. Technol., vol. 42, no. 4, pp. 641-646, Nov. 1993.

[20] P. Hande, S. Rangan, M. Chiang, and X. Wu, "Distributed uplink power control for optimal SIR assignment in cellular data networks," IEEE/ACM Trans. Netw., vol. 16, no. 6, pp. 1420-1433, Dec. 2008.

[21] N. Bambos, S. C. Chen, and G. J. Pottie, "Channel access algorithms with active link protection for wireless communication networks with power control," IEEE/ACM Trans. Netw., vol. 8, no. 5, pp. 583-597, Oct. 2000.

[22] A. Horn and A. Johnson, Matrix Analysis, 1st edition. Cambridge University Press, 1985.

[23] J. Mo and J. Walrand, "Fair end-to-end window-based congestion control," IEEE/ACM Trans. Netw., vol. 8, no. 5, pp. 556-567, Oct. 2000.

[24] J. Zander, "Performance of optimum transmitter power control in cellular radio systems," IEEE Trans. Veh. Technol., vol. 41, no. 1, pp. 57-62, Feb. 1992.

[25] H. Boche and S. Stanczak, "Convexity of some feasible QoS regions and asymptotic behavior of the minimum total power in CDMA systems," IEEE Trans. Commun., vol. 52, no. 12, pp. 2190-2197, Dec. 2004.

[26] D. P. Bertsekas, Nonlinear Programming, 2nd edition. Athena Scientific, 1999.

[27] E. Deutsch and M. Neumann, "Derivatives of the Perron root at an essentially nonnegative matrix and the group inverse of an M-matrix," J. Math. Anal. Applicat., vol. 102, no. 1, pp. 1-29, 1984. Available: http://www.sciencedirect.com/science/article/pii/0022247X84901987

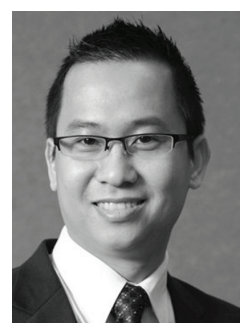

Duy Trong Ngo (Danny) (S'08) received the B.Eng. (with First-class Honours and the University Medal) degree in telecommunication engineering from the University of New South Wales, Sydney, NSW, Australia, in 2007, and the M.Sc. degree in electrical engineering (communication) from the University of Alberta, Edmonton, AB, Canada, in 2009. He is currently working toward the Ph.D. degree in electrical engineering with the Department of Electrical and Computer Engineering, McGill University, Montréal, QC, Canada. His research interest is in the area of radio resource allocation for wireless communications systems with special emphasis on heterogeneous networks.

From 2003 to 2006, Mr. Ngo was the recipient of the Australian Development Scholarship. He received the 2006 NICTA Telecommunications Excellence Award. The highest standing telecommunication engineering graduate, he was awarded the University Medal upon graduation at the University of New South Wales. During 2007-2009, he received the Alberta Ingenuity Fund Student Scholarship and the $i$ CORE ICT Graduate Student Award. He is currently the recipient of the Alexander Graham Bell Canada Graduate Scholarship, the McGill Engineering Doctoral Award, and the Clifford Pang Doctoral Fellowship.

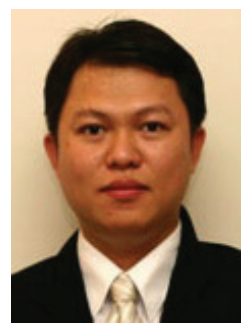

Long Bao Le (S'04-M'07-SM'12) received the B.Eng. (with Highest Distinction) degree from Ho Chi Minh City University of Technology, Vietnam, in 1999, the M.Eng. degree from Asian Institute of Technology, Pathumthani, Thailand, in 2002, and the Ph.D. degree from the University of Manitoba, Winnipeg, MB, Canada, in 2007.

From 2008 to 2010, he was a postdoctoral research associate with Massachusetts Institute of Technology, Cambridge, MA. Since 2010, he has been an assistant professor with the Institut National de la Recherche Scientifique (INRS), Université du Québec, Montréal, QC, Canada, where he leads a research group working on cognitive radio and dynamic spectrum sharing, radio resource management, network control and optimization.

Dr. Le is a member of the editorial board of IEEE COMMUNICATIONS SuRVEYS AND TUTORIALS and IEEE WIRELESS COMMUNICATIONS LETTERS. He has served as technical program committee co-chairs of the Wireless Networks track at IEEE VTC'2011-Fall and the Cognitive Radio and Spectrum Management track at IEEE PIMRC'2011.

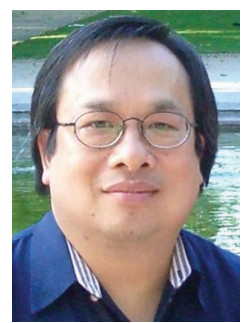

Tho Le-Ngoc (F'97) received the B.Eng. (with Distinction) degree in electrical engineering in 1976, the M.Eng. degree in microprocessor applications in 1978 from McGill University, Montréal, QC, Canada, and the Ph.D. degree in digital communications in 1983 from the University of Ottawa, Ottawa, ON, Canada.

From 1977 to 1982, he was with Spar Aerospace Limited, where he was involved in the development and design of satellite communications systems. From 1982 to 1985, he was an Engineering Manager of the Radio Group in the Department of Development Engineering of SRTelecom Inc., where he developed the new point-to-multipoint DA-TDMA/TDM Subscriber Radio System SR500. From 1985 to 2000, he was a Professor with the Department of Electrical and Computer Engineering, Concordia University, Montréal, QC, Canada. Since 2000, he has been a Professor with the Department of Electrical and Computer Engineering, McGill University, Montréal, QC, Canada. His research interest is in the area of broadband digital communications.

Dr. Le-Ngoc is a Senior Member of the Ordre des Ingénieurs du Québec and a Fellow of the Institute of Electrical and Electronics Engineers, the Engineering Institute of Canada, the Canadian Academy of Engineering, and the Royal Society of Canada. He is the recipient of the 2004 Canadian Award in Telecommunications Research, and the 2005 IEEE Canada Fessenden Award. He is the Canada Research Chair (Tier I) on Broadband Access Communications and the Bell Canada/NSERC Industrial Research Chair on Performance \& Resource Management in Broadband xDSL Access Networks. 